\title{
EXPANDING TRANSLATES OF CURVES AND DIRICHLET-MINKOWSKI THEOREM ON LINEAR FORMS
}

\author{
NIMISH A. SHAH
}

\section{INTRODUCTION}

Extending Dirichlet's theorem (1842) on simultaneous Diophantine approximation in various forms, Minkowski (1896) proved the following theorem as a consequence of his convex body theorem [15, Chapter II]:

Minkowski's theorem on linear forms. Let $\left(\varphi_{i j}\right) \in \mathrm{SL}(n, \mathbb{R})$ and $\alpha_{1}, \ldots, \alpha_{n}$ be positive numbers with $\alpha_{1} \cdots \alpha_{n}=1$. Then there exist integers $x_{1}, \ldots, x_{n}$, not all zero, such that

$$
\begin{aligned}
\left|\varphi_{11} x_{1}+\cdots+\varphi_{1 n} x_{n}\right| & \leq \alpha_{1}, \\
\left|\varphi_{i 1} x_{1}+\cdots+\varphi_{i n} x_{i n}\right| & <\alpha_{i} \quad(2 \leq i \leq n) .
\end{aligned}
$$

By putting $\varphi_{11}=\cdots=\varphi_{n n}=1$ and $\varphi_{i j}=0$ for $i \neq j$ and $i \geq 2$, we obtain a multiplicative variation of Dirichlet's theorem: Given $\left(\xi_{1}, \ldots, \xi_{k}\right) \in \mathbb{R}^{k}$ and positive integers $N_{1}, \ldots, N_{k}$, there exist integers $q_{1}, \ldots, q_{k}$ and $p$, not all zero, such that

$$
\left|q_{1} \xi_{1}+\cdots+q_{k} \xi_{k}-p\right| \leq\left(N_{1} \cdots N_{k}\right)^{-1}, \quad\left|q_{i}\right|<N_{i} \quad(1 \leq i \leq k) .
$$

Following Davenport and Schmidt 7], we say that given any infinite set $\mathcal{N} \subset \mathbb{N}^{k}$, Dirichlet's theorem (DT) cannot be improved along $\mathcal{N}$ for $\left(\xi_{1}, \ldots, \xi_{k}\right) \in \mathbb{R}^{k}$, if for every $0<\mu<1$ there are infinitely many $\left(N_{1}, \ldots, N_{k}\right) \in \mathcal{N}$ such that the following system of inequalities is insoluble for integers $q_{1}, \ldots, q_{k}$ and $p$, not all zero:

$$
\left|q_{1} \xi_{1}+\cdots+q_{k} \xi_{k}-p\right| \leq \mu\left(N_{1} \cdots N_{k}\right)^{-1}, \quad\left|q_{i}\right|<\mu N_{i} \quad(1 \leq j \leq k) .
$$

Davenport and Schmidt [7] showed that for $\mathcal{N}=\left\{(N, \ldots, N) \in \mathbb{Z}^{k}: N \in \mathbb{N}\right\}$, the DT cannot be improved along $\mathcal{N}$ for almost all points of $\mathbb{R}^{k}$. The same conclusion was obtained by Kleinbock and Weiss [11] for sets $\mathcal{N} \subset \mathbb{Z}^{k}$ with infinite projection on each coordinate.

In fact, Davenport and Schmidt [8] showed that for $k=2$ and for almost every $\xi \in$ $\mathbb{R}$, the inequalities (1.3) for $\left(\xi_{1}, \xi_{2}\right)=\left(\xi, \xi^{2}\right)$ do not have a nonzero integral solution for infinitely many $N_{1}=N_{2}$ and $\mu<\frac{1}{4}$. Such results for related quantities, say for points on a certain type of curve or a submanifold, were subsequently generalized in [1, 9, 2. More recently very general results on this problem were obtained in [1]

Received by the editors December 15, 2008.

2010 Mathematics Subject Classification. Primary 22E40, 11J83.

Key words and phrases. Flow on homogeneous spaces, geometry of numbers, Dirichlet's theorem, Minkowski's theorem, Diophantine approximation, unipotent flows, Ratner's theorem.

This research was supported in part by Swarnajayanti Fellowship.

(C)2009 American Mathematical Society Reverts to public domain 28 years from publication 
by Kleinbock and Weiss, who introduced the dynamical approach to this question and also considered the multiplicative version of the Dirichlet approximation. All of these non-improvability results were proved only for $\mu \leq \mu_{0}$ for some small explicit value of $\mu_{0}<1$ depending on the curve or the submanifold.

In the case of $\mathcal{N} \subset\left\{(N, \ldots, N) \in \mathbb{Z}^{k}: N \in \mathbb{N}\right\}$, in 20] it was shown that for any analytic curve which is not contained in a proper affine subspace of $\mathbb{R}^{k}$, the DT cannot be improved along $\mathcal{N}$ for almost all points on the curve, that is, for all $\mu<1$. In this article we will extend this result for any $\mathcal{N}$.

Theorem 1.1. Let $\mathcal{N}$ be an infinite subset of $\mathbb{N}^{k}$. Then for any analytic curve $\varphi:[a, b] \rightarrow \mathbb{R}^{k}$ whose image is not contained in a proper affine subspace, the DT cannot be improved along $\mathcal{N}$ for $\varphi(s)$ for Lebesgue almost every $s \in[a, b]$.

This theorem can be reformulated in terms of dynamics of flows on the homogeneous space $\operatorname{SL}(n, \mathbb{R}) / \mathrm{SL}(n, \mathbb{Z})$; cf. [11, $\S 2.1]$. We need to prove that certain sequences of expanding translates of a curve in this space tend to become uniformly distributed. To adapt the strategy of [20] for the general $\mathcal{N}$, we will need to overcome significant technical difficulties, whose resolution requires making new observations and developing much sharper methods.

1.1. Asymptotic equidistribution of translated curves. Let $n \geq 2$ and $G=$ $\mathrm{SL}(n, \mathbb{R})$. For $\boldsymbol{\tau}=\left(\tau_{1}, \ldots, \tau_{n-1}\right) \in \mathbb{R}^{n-1}$ and $\boldsymbol{\xi}=\left(\xi_{1}, \ldots, \xi_{n-1}\right) \in \mathbb{R}^{n-1}$, define

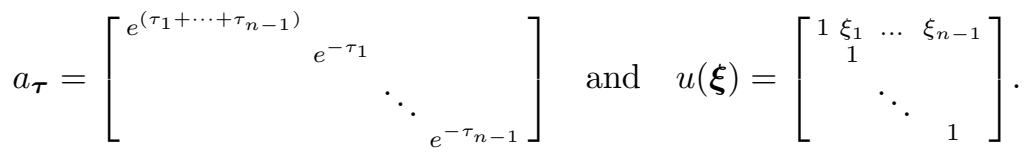

Let $\mathscr{T}=\left\{\boldsymbol{\tau}_{i}=\left(\tau_{i, 1}, \ldots, \tau_{i, n-1}\right)\right\}_{i \in \mathbb{N}} \subset \mathbb{R}^{n-1}$ be a sequence such that $\tau_{i, 1} \geq$ $\tau_{i, 2} \geq \cdots \geq \tau_{i, n} \geq 0$ for all $i$, and for some $1 \leq m_{1} \leq n-1$,

$$
\lim _{i \rightarrow \infty} \tau_{i, r}=+\infty \text { if } r \leq m_{1} \text { and } \lim _{i \rightarrow \infty} \tau_{i, r}=\tau(r)<\infty \text { if } r>m_{1}
$$

For $2 \leq m \leq n$, define

$$
Q_{m}=\left\{\left[\begin{array}{cc}
g & \boldsymbol{w} \\
\mathbf{0} & I_{n-m}
\end{array}\right] \in G: g \in \mathrm{SL}(m, \mathbb{R}), \boldsymbol{w} \in \mathrm{M}_{m \times(n-m)}(\mathbb{R})\right\},
$$

where $\mathbf{0}$ is the $(n-m) \times m$-zero matrix and $I_{n-m}$ is the $(n-m) \times(n-m)$-identity matrix.

The main goal of this article is to prove the following:

Theorem 1.2. Let $\varphi: I=[a, b] \rightarrow \mathbb{R}^{n-1}$ be an analytic map whose image is not contained in a proper affine subspace. Let $L$ be a Lie group and $\Lambda$ a lattice in $L$. Let $\rho: G \rightarrow L$ be a continuous homomorphism. Let $\mathscr{T}$ be a sequence in $\mathbb{R}^{k}$ and $1 \leq m_{1} \leq n-1$ be defined as above. Let $x_{0} \in L / \Lambda$ and $H$ be a minimal closed subgroup of $L$ containing $\rho\left(Q_{m_{1}+1}\right)$ such that the orbit $H x_{0}$ is closed and admits a unique $H$-invariant probability measure, say $\mu_{H}$. Then for any bounded continuous function $f$ on $L / \Lambda$ the following holds:

$$
\lim _{i \rightarrow \infty} \frac{1}{|b-a|} \int_{a}^{b} f\left(\rho\left(a_{\boldsymbol{\tau}_{i}} u(\varphi(s))\right) x_{0}\right) d s=\int_{H x_{0}} f\left(\rho\left(a_{\tau_{0}}\right) x_{0}\right) d \mu_{H}(x),
$$

where $\boldsymbol{\tau}_{0}=\left(0, \ldots, 0, \tau\left(m_{1}+1\right), \ldots, \tau(n-1)\right)$. 
Note that $\rho\left(Q_{m_{1}+1}\right)$ is generated by Ad-unipotent one-parameter subgroups of $L$. Hence by Ratner's theorem [14], $H x_{0}$ is the closure of the $\rho\left(Q_{m_{1}+1}\right)$-orbit of $x_{0}$. The above result in the case when $\boldsymbol{\tau}_{i}=\left(\tau_{i}, \ldots, \tau_{i}\right) \in \mathbb{R}^{n-1}$ for a sequence $\tau_{i} \stackrel{i \rightarrow \infty}{\longrightarrow} \infty$ was proved in $[20$. We will generalize that proof to obtain the above result. The main new contribution here is a strong general result about dynamics of intertwined linear actions of various $\mathrm{SL}(m, \mathbb{R})$ 's contained in $G$. Along with new interesting observations, its proof crucially uses the 'Basic lemma' from [20] on joint linear dynamics of various $\operatorname{SL}(2, \mathbb{R})$ 's contained in $\operatorname{SL}(n, \mathbb{R})$.

For the basic application of the theorem we will put $L=G, \Lambda=\operatorname{SL}(n, \mathbb{Z})$, $\rho$ the identity homomorphism, and $x_{0}=\operatorname{SL}(n, \mathbb{Z})$. Then $H=Q_{m_{1}+1}$, because $Q_{m_{1}+1} \cap \mathrm{SL}(n, \mathbb{Z})$ is a lattice in $Q_{m_{1}+1}$.

For more examples, let $\sigma$ be an involutive automorphism of $\operatorname{SL}(n, \mathbb{R})$ defined by

$$
\sigma(g):=\mathfrak{w}\left({ }^{\mathbf{t}} g^{-1}\right) \mathfrak{w}^{-1}, \quad \forall g \in \mathrm{SL}(n, \mathbb{R}),
$$

where $\mathfrak{w} \in \mathrm{GL}(n, \mathbb{R})$ permutes the standard basis $\left\{e_{1}, \ldots, e_{n}\right\}$ of $\mathbb{R}^{n}$ such that

$$
\mathfrak{w}\left(e_{i}\right)=e_{n+1-i}, \quad \forall 1 \leq i \leq n .
$$

Note that

$$
\begin{aligned}
& a_{\boldsymbol{\tau}}^{\prime}:=\sigma\left(a_{\boldsymbol{\tau}}\right)=\left[\begin{array}{llll}
e^{\tau_{n-1}} & & \\
& \ddots & \\
& & e^{\tau_{1}} & \\
& & & e^{-\left(\tau_{1}+\cdots+\tau_{n-1}\right)}
\end{array}\right],
\end{aligned}
$$

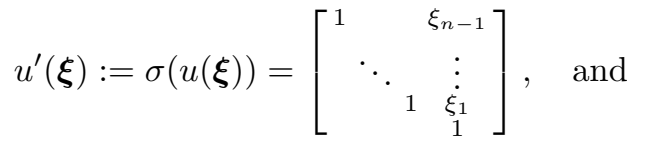

$$
\begin{aligned}
& Q_{m}^{\prime}:=\sigma\left(Q_{m}\right)=\left\{\left[\begin{array}{cc}
I_{n-m} & \boldsymbol{w} \\
\mathbf{0}_{m \times(n-m)} & g
\end{array}\right]: g \in \mathrm{SL}(m, \mathbb{R}), \boldsymbol{w} \in \mathrm{M}_{(n-m) \times m}(\mathbb{R})\right\} .
\end{aligned}
$$

Another application of Theorem 1.2 is obtained as follows: Let $L=G \times G$ and define the homomorphism $\rho: G \rightarrow L$ by

$$
\rho(g):=(g, \sigma(g)), \quad \forall g \in G .
$$

Let $\Lambda=\operatorname{SL}(n, \mathbb{Z}) \times \operatorname{SL}(n, \mathbb{Z})$. Then $\rho(\operatorname{SL}(n, \mathbb{Z})) \subset \Lambda$. Then $\rho\left(Q_{m_{1}+1}\right) \cap \Lambda$ is a lattice in $\rho\left(Q_{m_{1}+1}\right)$. Put $x_{0}=e \Lambda$. If we apply Theorem 1.2 in this case, then for its conclusion $H=\rho\left(Q_{m_{1}+1}\right)$.

1.2. Some applications. Using the conclusion of the theorem in the above example, we obtain the following result on non-improvability of Dirichlet's theorem on simultaneous Diophantine approximation in the dual form.

Theorem 1.3. Let $k \geq 1$, and let $\varphi: I=[a, b] \rightarrow \mathbb{R}^{k}$ be an analytic curve whose image is not contained in a proper affine subspace. Let $\mathcal{N}$ be an infinite subset of $\mathbb{N}^{k}$. Then for almost every $s \in I$ and any $\mu<1$, there exist infinitely many $\left(N_{1}, \ldots, N_{k}\right) \in \mathcal{N}$ such that both the following sets of inequalities are simultaneously insoluble:

$$
\left|q_{1} \varphi(s)+\cdots+q_{k} \varphi(s)-p\right| \leq \mu\left(N_{1} \ldots N_{k}\right)^{-1}, \quad\left|q_{i}\right| \leq \mu N_{i} \quad(1 \leq i \leq k)
$$

for $\left(p, q_{1}, \ldots, q_{k}\right) \in \mathbb{Z}^{k+1} \backslash\{\mathbf{0}\}$, and

$$
\left|q \varphi_{i}(s)-p_{i}\right| \leq \mu N_{i}^{-1} \quad(1 \leq i \leq k), \quad|q| \leq \mu N_{1} \ldots N_{k}
$$

for $\left(q, p_{1}, \ldots, p_{k}\right) \in \mathbb{Z}^{k+1} \backslash\{\mathbf{0}\}$. 
The above statement is stronger than Theorem [1.1] It also generalizes [20, Theorem 1.4], which considered the case of $\mathcal{N}$ where $N_{1}=N_{2}=\cdots=N_{k}$.

The following statement is an immediate consequence of Theorem 1.3 .

Corollary 1.4. Let $\mathcal{N} \subset \mathbb{N}^{n}$ be an infinite set. Let $M$ be a connected Riemannian analytic submanifold of $\mathbb{R}^{k}$ such that $M$ is not contained in a proper affine subspace of $\mathbb{R}^{k}$. We assume that $M$ can be measurably fibered by analytic curves which are not contained in proper affine subspaces of $\mathbb{R}^{k}$. Then with respect to the measure class on $M$ associated to the Riemannian volume form, for almost every $\boldsymbol{\xi} \in M$, the DT cannot be improved for $\boldsymbol{\xi}$ along $\mathcal{N}$.

In fact, the conclusion of Theorem 1.3 holds for almost all $\boldsymbol{\xi} \in M$ in place of $\varphi(s)$.

Remark 1.5. The conclusion of Corollary 1.4 holds without the additional condition on the manifold $M$ that $M$ can be measurably fibered by analytic curves not contained in proper affine subspaces. In order to prove this, we need the following extension of Theorem 1.2 for analytic maps of several variables: Let $I=[0,1]^{d} \subset \mathbb{R}^{d}$, and let $\varphi: I \rightarrow \mathbb{R}^{k}$ be an analytic map such that $\varphi(I)$ is not contained in a proper affine subspace of $\mathbb{R}^{k}$. Then the conclusion (1.6) of Theorem 1.2 is valid if we integrate over the box I with respect to the Lebesgue measure, instead of over the interval $[a, b]$.

This statement can be proved by the method of this article using generalized versions of Proposition 3.3 and Proposition 6.2 for higher-dimensional maps. The generalized propositions can be obtained by combining the techniques from [10] and [17.

It may be noted that in the above results we can take $\mathcal{N}$ with bounded projections on some of the coordinates. In this case the non-improvability results were not known earlier even for almost all points of $\mathbb{R}^{k}$. It is also natural to consider sequences $\mathcal{N}$ in $\left(\mathbb{R}_{>0}\right)^{k}$ rather than in $\mathbb{N}^{k}$. In that case we have the following result.

Corollary 1.6. The conclusions of Theorem 1.3 and Corollary 1.4 are valid for any unbounded sequence $\mathcal{N} \subset\left(\mathbb{R}_{>0}\right)^{k}$ satisfying the condition that none of the coordinate projections of this sequence has a limit point in $\mathbb{R} \backslash \mathbb{N}$.

To deduce this from Theorem 1.3, we replace the vectors in $\mathcal{N}$ by integral vectors whose coordinates are nearest integers of the original coordinates. Then under the given conditions, the ratios of their corresponding coordinates converge to 1 in the limit. Given $\mu<1$, we choose $1>\mu_{1}>\mu$. Now the product of these ratios $(<1)$ times $\mu_{1}$ is bigger than $\mu$ for all but finitely many elements of these sequences.

The condition on limit points of coordinates of $\mathcal{N}$ in Corollary [1.6 is also a necessary condition:

Theorem 1.7. Let $\mathcal{N} \subset\left(\mathbb{R}_{>0}\right)^{k}$ be an unbounded sequence such that one of the coordinates converges to an element of $\mathbb{R} \backslash \mathbb{N}$. Then there exists a positive $\mu<1$ such that for all but finitely many $\left(N_{1}, \ldots, N_{k}\right) \in \mathcal{N}$ and every $\boldsymbol{\xi} \in \mathbb{R}^{k}$, the system of inequalities (1.3) admits nonzero integral solutions.

This result is a consequence of a theorem due to Minkowski and Hajós on critical lattices. In fact, the result generalizes a counterexample given in [11, §4.4], and combined with Corollary 1.6, it answers a question raised there. 
1.3. Non-improvability of Minkowski's theorem under different conditions. In the above discussion, we considered a special case of Minkowski's linear forms theorem to obtain the multiplicative version of Dirichlet's approximation theorem. Now we consider different restrictions in Minkowski's theorem and the corresponding non-improvability statements. The following result is a direct consequence of [20, Theorem 1.8]:

Theorem 1.8. Let $\varphi=\left(\varphi_{i j}\right): I=[a, b] \rightarrow \mathrm{SL}(n, \mathbb{R})$ be an analytic map such that $\mathbb{R}-\operatorname{span}\left\{\varphi_{1, j}(s): s \in I\right\}=\mathbb{R}^{n}$. Let $\mathscr{N}$ be an infinite set of positive integers. Then for almost every $s \in I$, there exists an infinite subset $\mathscr{N}_{s} \subset \mathscr{N}$ such that for any $\mu<1$ the following system of inequalities is insoluble in $\left(x_{1}, \ldots, x_{n}\right) \in \mathbb{Z}^{n} \backslash\{0\}$ for any $N \in \mathscr{N}_{s}$ :

$$
\begin{aligned}
\left|\varphi_{1,1}(s) x_{1}+\cdots+\varphi_{1, n}(s) x_{n}\right| & \leq \mu N^{-n} \\
\left|\varphi_{i, 1}(s) x_{1}+\cdots+\varphi_{i, n}(s) x_{i, n}\right| & \leq \mu N \quad(2 \leq i \leq n) .
\end{aligned}
$$

Now we ask whether we can obtain a multiplicative version of the above statement. In this direction we can prove the special case, which also generalizes Theorem 1.1 .

Theorem 1.9. Let $\mathcal{N}$ be an infinite subset of $\left(\mathbb{R}_{>0}\right)^{n-1}$ such that its projection on each coordinate has no limit point. Let $\varphi$ be as in Theorem 1.8. We further assume that $\varphi_{i 1}(s) \equiv 0$ and $\varphi_{i j}(s) \equiv \varphi_{i j}$ are constant functions for all $i \geq 2$. Then for almost every $s \in I$, there exists an infinite subset $\mathcal{N}_{s} \subset \mathcal{N}$ such that for every $\mu<1$ the following system of inequalities is insoluble in $\left(x_{1}, \ldots, x_{n}\right) \in \mathbb{Z}^{n} \backslash\{0\}$ for any $\left(N_{1}, \ldots, N_{n-1}\right) \in \mathcal{N}_{s}$ :

$$
\begin{aligned}
\left|\varphi_{11}(s) x_{1}+\cdots+\varphi_{1 n}(s) x_{n}\right| & \leq \mu\left(N_{1} \ldots N_{n-1}\right)^{-1}, \\
\left|\varphi_{i 2} x_{2}+\cdots+\varphi_{i n} x_{n}\right| & \leq \mu N_{i-1} \quad(2 \leq i \leq n) .
\end{aligned}
$$

It is a question of whether the conditions on $\varphi_{i, j}(s)$ can be removed for $i \geq 2$.

1.4. Uniform versions of the equidistribution statement. Let the notation be as in 1.1

Theorem 1.10. Let $\varphi: I=[a, b] \rightarrow \mathbb{R}^{n-1}$ be an analytic map whose image is not contained in a proper affine subspace. Let $L$ be a Lie group and let $\Gamma$ be a lattice in L. Let $\rho: G \rightarrow L$ be a continuous homomorphism. Let $\mathscr{T}=\left\{\boldsymbol{\tau}_{i}\right\}$ be a sequence as in Theorem 1.2. Let $x_{0} \in L / \Lambda$ be such that $\rho\left(Q_{m_{1}+1}\right) x_{0}$ is dense in $L / \Lambda$. Let $x_{i} \stackrel{i \rightarrow \infty}{\longrightarrow} x_{0}$ be a convergent sequence in $L / \Lambda$. Then for any bounded continuous function $f$ on $L / \Lambda$

$$
\lim _{i \rightarrow \infty} \frac{1}{|b-a|} \int_{a}^{b} f\left(\rho\left(a_{\boldsymbol{\tau}_{i}} u(\varphi(s))\right) x_{i}\right) d s=\int_{L / \Lambda} f d \mu_{L},
$$

where $\mu_{L}$ is the unique $L$-invariant probability measure on $L / \Lambda$.

For the special case of $L=G, \rho$ the identity map, and $m_{1}=n-1$, that is, $Q_{m_{1}+1}=G$, we can take any convergent sequence $x_{i} \rightarrow x_{0}$ in the above theorem.

A more general uniform version is as follows.

Theorem 1.11. Let the notation be as in Theorem 1.10. Let $\mathcal{K}$ be a compact subset of $L / \Lambda$. Then given $\epsilon>0$ and a bounded continuous function $f$ on $L / \Lambda$, there exist 
finitely many proper closed subgroups $H_{1}, \ldots, H_{r}$ of $L$ such that for each $1 \leq i \leq r$, $H_{i} \cap \Lambda$ is a lattice in $H_{i}$ and there exists a compact set

$$
C_{i} \subset N\left(H_{i}, \rho\left(Q_{m_{1}+1}\right)\right):=\left\{g \in L: \rho\left(Q_{m_{1}+1}\right) g \subset g H_{i}\right\}
$$

such that the following holds: Given any compact set

$$
F \subset \mathcal{K} \backslash \bigcup_{i=1}^{r} C_{i} \Lambda / \Lambda
$$

there exists $i_{0}>0$ such that for any $x \in F$ and any $i \geq i_{0}$,

$$
\left|\frac{1}{b-a} \int_{a}^{b} f\left(\rho\left(a_{\boldsymbol{\tau}_{i}} u(\varphi(s))\right) x\right) d s-\int_{L / \Lambda} f d \mu_{L}\right|<\epsilon .
$$

Both of the above results in the special case, when for each $i$ all coordinates of $\boldsymbol{\tau}_{i}$ are same, were obtained earlier in $[20, \S 1.2]$.

\section{Deduction of Theorem 1.3 from Theorem 1.2}

We express $\mathcal{N} \subset \mathbb{N}^{k}$ as a sequence

$$
\mathcal{N}=\left\{\left(N_{i, 1}, N_{i, 2}, \ldots, N_{i, k}\right) \in \mathbb{N}^{k}: i \in \mathbb{N}\right\} .
$$

Note that the conclusions of Theorem 1.3 does not depend on a fixed permutation of the coordinates. Since there are at most $k$ ! permutations possible, by passing to a subsequence of $\mathcal{N}$ and applying a permutation, without loss of generality we may assume that

$$
N_{i, 1} \geq N_{i, 2} \geq \cdots \geq N_{i, k}, \quad \forall i \in \mathbb{N} .
$$

Since $\mathcal{N}$ is infinite, by further passing to a subsequence, we may assume that there exists $m_{1} \geq 1$ such that $N_{i, m_{1}} \stackrel{i \rightarrow \infty}{\longrightarrow} \infty$, and for each $m_{1}<j \leq k$ there exists $N_{0, j} \in \mathbb{N}$ such that $N_{i, j}=N_{0, j}$ for every $i \in \mathbb{N}$. We define

$$
\boldsymbol{\tau}_{i}=\left(\log N_{i, 1}, \ldots, \log N_{i, k}\right) \in\left(\mathbb{R}_{\geq 0}\right)^{k}, \quad \forall i \in \mathbb{N},
$$

and put $\mathscr{T}=\left(\boldsymbol{\tau}_{i}\right)_{i \in \mathbb{N}}$. Then $\mathscr{T}$ satisfies the conditions of $\$ 1.1$ We put

$$
\boldsymbol{\tau}_{0}:=\left(0, \ldots, 0, \log N_{0, m_{1}+1}, \ldots, \log N_{0, k}\right) .
$$

Let $n=k+1$. We identify the space $\Omega$ of unimodular lattices in $\mathbb{R}^{n}$ with $\operatorname{SL}(n, \mathbb{R}) / \operatorname{SL}(n, \mathbb{Z})$. Given $0<\mu<1$, we define

$$
\begin{aligned}
& B_{\mu}=\left\{\left(\xi_{1}, \ldots, \xi_{n}\right) \in \mathbb{R}^{n}: \sup _{1 \leq i \leq n}\left|\xi_{i}\right| \leq \mu\right\}, \\
& K_{\mu}=\left\{\Delta \in \Omega: \Delta \cap B_{\mu}=\{0\}\right\} .
\end{aligned}
$$

For $\left(N_{1}, \ldots, N_{k}\right) \in \mathcal{N}$, let $\boldsymbol{\tau}=\left(\log N_{1}, \ldots, \log N_{k}\right)$. Then for any $s \in I, \boldsymbol{x}=$ $\left(p, q_{1}, \ldots, q_{k}\right) \in \mathbb{Z}^{n}$, and $\boldsymbol{x}^{\prime}=\left(p_{k}, \ldots, p_{1}, q\right) \in \mathbb{Z}^{k}$, we have

$$
\rho\left(a_{\boldsymbol{\tau}} u(\varphi(s))\right)\left(\boldsymbol{x}, \boldsymbol{x}^{\prime}\right)=\left(\left(\begin{array}{c}
\left(N_{1} \cdots N_{k}\right)\left(p+\sum_{i=1}^{k} q_{i} \varphi_{i}(s)\right) \\
N_{1}^{-1} q_{1} \\
\vdots \\
N_{k}^{-1} q_{k}
\end{array}\right),\left(\begin{array}{c}
N_{k}\left(q \varphi_{k}(s)+p_{k}\right) \\
\vdots \\
N_{1}\left(q \varphi_{1}(s)+p_{1}\right) \\
\left(N_{1} \cdots N_{k}\right)^{-1} q
\end{array}\right)\right) .
$$

Therefore (cf. [11, §2.1], [20, §2])

$$
\begin{aligned}
& \text { (1.11) is soluble } \Longleftrightarrow a_{\boldsymbol{\tau}} u(\varphi(s)) \boldsymbol{x} \in B_{\mu} \Longleftrightarrow a_{\boldsymbol{\tau}} u(\varphi(s)) \mathbb{Z}^{n} \notin K_{\mu}, \\
& \text { (1.12) is soluble } \Longleftrightarrow a_{\boldsymbol{\tau}}^{\prime} u^{\prime}(\varphi(s)) \boldsymbol{x}^{\prime} \in B_{\mu} \Longleftrightarrow a_{\tau}^{\prime} u^{\prime}(\varphi(s)) \mathbb{Z}^{n} \notin K_{\mu} .
\end{aligned}
$$


Hence Theorem 1.3 will be proved if we prove the following:

Proposition 2.1. Put $x_{0}=$ : $\left(\mathbb{Z}^{n}, \mathbb{Z}^{n}\right) \in \Omega \times \Omega$. Given $\mu<1$, define

$$
E_{\mu}:=\left\{s \in[a, b]: \rho\left(a_{\boldsymbol{\tau}_{i}} u(\varphi(s))\right) x_{0} \notin K_{\mu} \times K_{\mu} \text { for all large } i\right\} .
$$

Then $\left|E_{\mu}\right|=0$, where $|\cdot|$ denotes the Lebesgue measure.

In order to deduce this proposition from Theorem 1.2, we need the following result, especially when $m_{1}<n-1=k$.

We define a compact set

$$
K_{1}=\bigcap_{0<\mu<1} K_{\mu}=\left\{\Delta \in \Omega: \sup _{1 \leq i \leq n}\left|\xi_{i}\right| \geq 1, \forall\left(\xi_{1}, \ldots, \xi_{n}\right) \in \Delta \backslash\{0\}\right\} .
$$

Theorem 2.2. $\rho\left(a_{\tau_{0}} Q_{m_{1}+1}\right) x_{0} \cap\left(K_{1} \times K_{1}\right) \neq \emptyset$.

To prove this, we will need the easier inclusion $(\supset)$ part of the following fact, which was guessed by Minkowski (1896) and proved by Hajós in 1941; see [3, XI.1.3]. Its full strength will be used later for proving Theorem 1.7.

Theorem 2.3. Let $N$ denotes the group of upper triangular unipotent matrices in $\mathrm{SL}(n, \mathbb{R})$. Let $\mathcal{W}_{n}=\left\{w \in \mathrm{GL}(n, \mathbb{Z}): w\right.$ permutes the standard basis of $\left.\mathbb{R}^{n}\right\}$. Then

$$
K_{1}=\bigcup_{w \in \mathcal{W}_{n}}\left(w N w^{-1}\right) \mathbb{Z}^{n}
$$

Proposition 2.4. Let $N^{-}$be the lower triangular unipotent subgroup of $\mathrm{SL}(n, \mathbb{R})$ and $\Gamma=\mathrm{SL}(n, \mathbb{Z})$. Let $\left(N_{1}, \ldots, N_{n-1}\right) \in \mathbb{N}^{n-1}$ and $\boldsymbol{\tau}:=\left(\log N_{1}, \ldots, \log N_{n-1}\right)$. Then

$$
a_{\tau} Q_{1} \Gamma \cap N^{-} \Gamma \neq \emptyset .
$$

Proof. For the involutive automorphism $\sigma$ as defined by (1.7), we have $\sigma\left(N^{-}\right)=$ $N^{-}, \sigma(\Gamma)=\Gamma, \sigma\left(a_{\boldsymbol{\tau}}\right)=a_{\boldsymbol{\tau}}^{\prime}$, and $\sigma\left(Q_{1}\right)=Q_{1}^{\prime}$. Therefore it is enough to prove that

$$
a_{\tau}^{\prime} Q_{1}^{\prime} \cap N^{-} \Gamma \neq \emptyset .
$$

We write $k=n-1$. Note that

$$
a_{\boldsymbol{\tau}}^{\prime} Q_{1}^{\prime}=\left\{\left[\begin{array}{cccc}
N_{k} & & & \xi_{k} \\
& \ddots & & \vdots \\
& & N_{1} & \xi_{1} \\
& & & \left(N_{1} \cdots N_{k}\right)^{-1}
\end{array}\right]: \xi_{i} \in \mathbb{R}\right\} .
$$

We define

$$
\gamma=\left[\begin{array}{ccccc}
N_{k} & & & & 1 \\
N_{k}-1 & N_{k-1} & & & 1 \\
N_{k}-1 & N_{k-1}-1 & \ddots & & \vdots \\
\vdots & \vdots & \vdots & N_{1} & 1 \\
N_{k}-1 & N_{k-1}-1 & \cdots & N_{1}-1 & 1
\end{array}\right] \in \operatorname{SL}(n, \mathbb{Z}) .
$$

We express the matrix $\gamma$ as a sum of a lower triangular matrix $L$ of determinant 1 and a matrix $M$ with only the last column nonzero. Then we choose $h \in N^{-}$such that $h L=\operatorname{diag}\left(N_{1}, \ldots, N_{k},\left(N_{1} \cdots N_{k}\right)^{-1}\right)$. Also $h M$ is a matrix with only the last column nonzero. In particular, $h \gamma=(h L+h M)$ is an upper triangular matrix of determinant 1. Hence $h \gamma \in a_{\tau}^{\prime} Q_{1}^{\prime}$ by (2.12). This proves (2.11). 
Proof of Theorem 2.2. Since $Q_{1} \subset Q_{m_{1}+1}$, by Proposition 2.4 there exist $g \in$ $Q_{m_{1}+1}, h \in N^{-}$, and $\gamma \in \Gamma$ such that $a_{\boldsymbol{\tau}_{0}} g=h \gamma$. Therefore $\rho\left(a_{\boldsymbol{\tau}_{0}} g\right)=\rho(h) \rho(\gamma)$. Now $\rho(\gamma) x_{0}=x_{0}$ and $\mathfrak{w} N \mathfrak{w}^{-1}=N^{-}$for $\mathfrak{w} \in \mathcal{W}_{n}$ as in (1.8). Therefore $\rho\left(a_{\boldsymbol{\tau}_{0}} g\right) x_{0}=$ $\rho(h) x_{0} \in K_{1} \times K_{1}$ by Theorem 2.3 .

Proof of Proposition 2.1. Let $\rho$ be as in (1.10). Then the orbit $\rho\left(Q_{m_{1}+1}\right) x_{0}$ is closed and admits a unique $\rho\left(Q_{m_{1}+1}\right)$-invariant probability measure, say $\lambda$. Fix $\mu<1$. Then $K_{\mu} \times K_{\mu}$ contains an open neighbourhood of $K_{1} \times K_{1}$. Therefore by Theorem 2.2. $K_{\mu} \times K_{\mu}$ contains a nonempty open subset of $\rho\left(a_{\boldsymbol{\tau}_{0}} Q_{m_{1}+1}\right) x_{0}$. Therefore there exists $\epsilon>0$ such that

$$
\lambda\left(\rho\left(a_{\boldsymbol{\tau}_{0}}\right)^{-1}\left(K_{\mu} \times K_{\mu}\right)\right)>\epsilon .
$$

So there exists $f \in \mathrm{C}_{\mathrm{c}}(L / \Lambda)$ such that $0 \leq f \leq 1, \operatorname{supp}(f) \subset K_{\mu} \times K_{\mu}$, and

$$
\int_{x \in \rho\left(Q_{m_{1}+1}\right) x_{0}} f\left(\rho\left(a_{\tau_{0}}\right) x\right) d \lambda(x) \geq \epsilon / 2 .
$$

Let $J$ be any subinterval of $[a, b]$ with nonempty interior. Then by Theorem 1.2 there exists $i_{0} \in \mathbb{N}$ such that for all $i \geq i_{0}$, we have

$$
\frac{1}{|J|} \int_{J} f\left(\rho\left(a_{\boldsymbol{\tau}_{i}} u(\varphi(s))\right) x_{0}\right) d s \geq \int f\left(\rho\left(a_{\boldsymbol{\tau}_{0}}\right) x\right) d \lambda(x)-\epsilon / 4 \geq \epsilon / 4 .
$$

Therefore by combining the definition of $E_{\mu}$ and the choice of $f$, we conclude that $|E \cap J| \leq(1-\epsilon / 4)|J|$. Since $J$ is an arbitrary open subinterval of $I$, by the Lebesgue density theorem $\left|E_{\mu}\right|=0$.

Thus we have completed the deduction of Theorem 1.3 .

Proof of Theorem 1.8. The result follows by the arguments as above. We need to consider only the first factor in (2.5) and $Q_{m_{1}+1}=G$. Clearly $K_{\mu}$ has strictly positive measure on $G / \Gamma$. The only difference is that to conclude (2.16) we need to use [20, Theorem 1.8], where the same conclusion as that of Theorem 1.2 was obtained for the given map $\varphi$ and $\boldsymbol{\tau}_{i}=\left(\tau_{i}, \ldots, \tau_{i}\right) \in \mathbb{R}^{n-1}$ for each $i$ such that $\tau_{i} \stackrel{i \rightarrow \infty}{\longrightarrow} \infty$.

Proof of Theorem 1.9, Again the deduction of this result is as above. One only considers the first factor. Here by our choice $Q_{m_{1}+1}=G$ and $K_{\mu}$ has strictly positive measure on $G / \Gamma$. Now note that there exist $g_{0} \in G$ and an analytic curve $\psi: I \rightarrow \mathbb{R}^{n-1}$ such that $\varphi(s)=u(\psi(s)) g_{0}$ for all $s \in I$. By our condition, the image of $\psi$ is not contained in any proper affine subspace of $\mathbb{R}^{n-1}$. We then apply Theorem 1.2 for $x_{0}=g_{0} \mathbb{Z}^{n}$.

Proof of Theorem 1.7. We write $\mathcal{N}=\left\{\left(N_{i, 1}, N_{i, 2}, \ldots, N_{i, k}\right) \in\left(\mathbb{R}_{>0}\right)^{k}: i \in \mathbb{N}\right\}$. Since there is no loss of generality if we permute the coordinates, we may assume that $N_{i, k} \stackrel{i \rightarrow \infty}{\longrightarrow} N_{0, k}$ and $N_{0, k} \notin \mathbb{N}$. Let $n=k+1$ and $x_{0}=\mathbb{Z}^{n} \in \Omega$. Let $a_{i}=a_{\left(0, \ldots, 0, \log N_{i, k}\right)}$ for all $i$. Then by (1.5),$a_{\boldsymbol{\tau}_{i}} Q_{k}=a_{i} Q_{k}$ for all $i$. Since $u(\boldsymbol{\xi}) \in Q_{k}$ for any $\boldsymbol{\xi} \in \mathbb{R}^{k}$, in view of (2.5), considering only the first factor, it is enough to show that there exist $0<\mu<1$ and $i_{0} \in \mathbb{N}$ such that

$$
a_{i} Q_{k} x_{0} \cap K_{\mu}=\emptyset, \quad \forall i \geq i_{0} .
$$

If (2.17) fails to hold, then there exists a sequence $i_{j} \in \mathbb{N}, \mu_{j}<1$, and $g_{j} \in Q_{k}$ such that $y_{j}:=a_{i_{j}} g_{j} x_{0} \in a_{i} Q_{k} x_{0} \cap K_{\mu_{j}}$ and $\mu_{j} \rightarrow 1$. Now any $\overline{K_{\mu_{j_{1}}}}$ is compact 
and contains $\overline{K_{\mu_{2}}}$ for every $j_{2}>j_{1}$, and $K_{1}=\bigcap_{j \in \mathbb{N}} \overline{K_{\mu_{j}}}$. Therefore after passing to a subsequence, $y_{j} \rightarrow y_{0}$ for some $y_{0} \in K_{1}$.

First we consider the case of $N_{0, k} \neq 0$. Let $a_{0}=a_{\left(0, \ldots, \log N_{0, k}\right)} \in G$. Then $a_{i} \rightarrow a_{0}$ as $i \rightarrow \infty$. Since $Q_{k} x_{0}$ is closed, we conclude that $y_{0} \in a_{0} Q_{k} x_{0} \cap K_{1}$.

Thus to prove (2.17), it is enough to show that

$$
a_{0} Q_{k} x_{0} \cap K_{1}=\emptyset .
$$

Suppose this intersection is nonempty. Then by Theorem 2.3

$$
a_{0} Q_{k} \cap \mathcal{W}_{n} N \Gamma \neq \emptyset \text {. }
$$

Applying $\sigma$ on both sets (see (1.7) and (1.9)), we get

$$
\sigma\left(a_{0}\right) Q_{k}^{\prime} \cap \mathcal{W}_{n} N \Gamma \neq \emptyset \text {. }
$$

Let $\left\{e_{1}, \ldots, e_{n}\right\}$ denote the standard basis of $\mathbb{R}^{n}$. Since $Q_{k}^{\prime} e_{1}=e_{1}$ and $\sigma\left(a_{0}\right) e_{1}=$ $N_{0, k} e_{1}$, there exist $\boldsymbol{v}=q_{1} e_{1}+\cdots+q_{n} e_{n} \in \Gamma e_{1} \subset \mathbb{Z}^{n}, w \in \mathcal{W}_{n}$, and $g=\left(x_{i j}\right) \in N$ such that

$$
N_{0, k} e_{1}=w g \boldsymbol{v} .
$$

Let $1 \leq r \leq n$ be such that $w^{-1} e_{1}=e_{r}$. Then we have

$$
\begin{aligned}
N_{0, k} & =q_{r}+\sum_{j>r} x_{i j} q_{j}, \\
0 & =q_{i}+\sum_{j>i} x_{i j} q_{j} \quad(i>r) .
\end{aligned}
$$

Putting $i=n$ in (2.23), we get that $q_{n}=0$. Now for any $i_{0}>r$, if $q_{j}=0$ for all $j>i_{0}$, then putting $i=i_{0}$ in (2.23), we get that $q_{i_{0}}=0$. Therefore by induction $q_{j}=0$ for all $j>r$. Therefore (2.22) becomes $N_{0, k}=q_{r} \in \mathbb{Z}$, a contradiction because $N_{0, k} \geq 0$ and $N_{0, k} \notin \mathbb{N} \cup\{0\}$ by our assumption. This shows that the intersection in (2.18) cannot be nonempty. Hence (2.17) must hold, and the proof is complete in the case when $N_{0, k} \neq 0$.

Now we consider the case of $N_{0, k}=0$. Since $a_{i} g_{i} x_{0} \rightarrow y_{0}$ as $i \rightarrow \infty$, there exists $\gamma_{i} \in \Gamma$ such that

$$
a_{i} g_{i} \gamma_{i} \rightarrow g_{0}, \quad \text { for some } g_{0} \in G \text { as } i \rightarrow \infty .
$$

Let $x_{i}=\sigma\left(\gamma_{i}^{-1}\right) e_{1} \in \mathbb{Z}^{n} \backslash\{0\}$ for each $i$. Then $\sigma\left(a_{i} g_{i} \gamma_{i}\right) x_{i}=N_{i, k} e_{1} \rightarrow N_{0, k} e_{1}=0$ as $i \rightarrow \infty$. This contradicts (2.24). This completes the proof that (2.17) holds in all cases.

Now we begin the proof of the main theorem, Theorem 1.2

\section{Nondivergence of translates}

Let the notation be as in $\$ 1.1$ and the statement of Theorem 1.2. We consider the action of $G$ on $L / \Lambda$ via the homomorphism $\rho$; that is, for any $x \in L / \Lambda$ and $g \in G$, we have $g x:=\rho(g) x$. Let $\left\{x_{i}\right\}_{i \in \mathbb{N}}$ be a sequence in $L / \Lambda$ such that $x_{i} \stackrel{i \rightarrow \infty}{\longrightarrow} x_{0}$. For any $i \in \mathbb{N}$ define $\mu_{i}$ to be the probability measure on $L / \Lambda$ as

$$
\int_{L / \Lambda} f d \mu_{i}:=\frac{1}{|I|} \int_{I} f\left(a_{\boldsymbol{\tau}_{i}} u(\varphi(s)) x_{i}\right) d s, \quad \forall f \in \mathrm{C}_{\mathrm{c}}(L / \Lambda) .
$$

Theorem 3.1. Given $\epsilon>0$, there exists a compact set $\mathcal{F} \subset L / \Lambda$ such that $\mu_{i}(\mathcal{F}) \geq$ $1-\epsilon$ for all large $i \in \mathbb{N}$. 
In the case of $L=\mathrm{SL}(n, \mathbb{R}), \rho$ the identity map, and $\Lambda=\mathrm{SL}(n, \mathbb{Z})$, the result was obtained by Kleinbock and Margulis [10.

3.1. Let $\mathscr{H}$ denote the collection of analytic subgroups $H$ of $G$ such that $H \cap \Lambda$ is a lattice in $H$ and a unipotent one-parameter subgroup of $H$ acts ergodically with respect to the $H$-invariant probability measure on $H / H \cap \Lambda$. Then $\mathscr{H}$ is a countable collection [13, 16].

Let $\mathfrak{l}$ denote the Lie algebra of $L$. Let $V=\bigoplus_{d=1}^{\operatorname{dim} \mathfrak{l}} \wedge^{d} \mathfrak{l}$ and consider the $\left(\bigoplus_{d=1}^{\operatorname{dim} \mathfrak{l}} \wedge^{d} \mathrm{Ad}\right)$-action of $L$ on $V$. Given $H \in \mathscr{H}$, let $\mathfrak{h}$ denote its Lie algebra, and fix $p_{H} \in \bigwedge^{\operatorname{dim} \mathfrak{h}} \mathfrak{h} \backslash\{0\} \subset V$. Let $\mathrm{N}_{\mathrm{L}}(H)$ denote the normalizer of $H$ in $L$. Then

$$
\operatorname{Stab}_{L}\left(p_{H}\right)=\mathrm{N}_{\mathrm{L}}^{1}(H):=\left\{g \in \mathrm{N}_{\mathrm{L}}(H): \operatorname{det}\left(\left.(\operatorname{Ad} g)\right|_{\mathfrak{h}}\right)=1\right\} .
$$

Proposition $3.2([6])$. The orbit $\Lambda \cdot p_{H}$ is a discrete subset of $V$.

Consider the $G$ action on $V$ via $\rho$; that is, $g v=\rho(g) v$ for all $g \in G$ and $v \in V$. Given a sequence $\mathscr{T}$ as in $\$ 1.1$, we define

$$
\begin{aligned}
& V_{\mathscr{T}}^{-}=\left\{v \in V: a_{\boldsymbol{\tau}_{i}} v \stackrel{i \rightarrow \infty}{\longrightarrow} 0\right\}, \quad V_{\mathscr{T}}^{+}=\left\{v \in V: a_{\boldsymbol{\tau}_{i}}^{-1} v \stackrel{i \rightarrow \infty}{\longrightarrow} 0\right\}, \\
& V_{\mathscr{T}}^{0}=\left\{v \in V: a_{\boldsymbol{\tau}_{i}} v \stackrel{i \rightarrow \infty}{\longrightarrow} v_{1} \text { and } a_{\boldsymbol{\tau}_{i}}^{-1} v \stackrel{i \rightarrow \infty}{\longrightarrow} v_{2} \text { for some } v_{1}, v_{2} \in V\right\} .
\end{aligned}
$$

Since $\left\{a_{\boldsymbol{\tau}}: \boldsymbol{\tau} \in \mathbb{R}^{n-1}\right\}$ acts on $V$ by $\mathbb{R}$-diagonalizable commuting automorphisms, by passing to a subsequence of $\mathscr{T}$, we have

$$
V=V_{\mathscr{T}}^{-} \oplus V_{\mathscr{T}}^{0} \oplus V_{\mathscr{T}}^{+} .
$$

Let $\pi_{0}^{\mathscr{T}}: V \rightarrow V_{\mathscr{T}}^{0}$ denote the corresponding projection.

3.2. Margulis-Dani nondivergence criterion. We recall the following criterion based on [5, 18, 10. Here we use the information that $\varphi$ is an analytic map.

Proposition 3.3 ([20, Proposition 3.4]). There exists a finite collection $\mathscr{W} \subset \mathscr{H}$ (depending only on $L$ and $\Lambda$ ) such that the following holds: Given $\epsilon>0$ and $R>0$, there exists a compact set $\mathcal{F} \subset L / \Lambda$ such that for any $h_{1}, h_{2} \in L$ and a subinterval $J \subset I$, one of the following conditions is satisfied:

(I) There exists $\gamma \in \Lambda$ and $W \in \mathscr{W}$ such that

$$
\sup _{s \in J}\left\|h_{1} u(\varphi(s)) h_{2} p_{W}\right\|<R .
$$

(II) $\frac{1}{|J|}\left|\left\{s \in J: h_{1} u(\varphi(s)) h_{2} \Lambda / \Lambda \in \mathcal{F}\right\}\right| \geq 1-\epsilon$.

In view of the above nondivergence criterion, it will follow that the main new ingredient in the proof of Theorem 3.1 is the following linear dynamical lemma, which is at the core of this article and is proved in the next section (see Lemma 4.1 and Corollary 4.10).

Lemma 3.4. For any finite-dimensional linear representation $V$ of $G$, any $v \in$ $V \backslash\{0\}$, and any $\mathcal{B} \subset \mathbb{R}^{n-1}$ not contained in a proper affine subspace of $\mathbb{R}^{n-1}$,

$$
\text { if } u(e) v \in V_{\mathscr{T}}^{-}+V_{\mathscr{T}}^{0} \text { for all } e \in \mathcal{B} \text {, then } \pi_{0}^{\mathscr{T}}(u(e) v) \neq 0 \text { for all } e \in \mathcal{B} \text {. }
$$


Proof of Theorem 3.1. Let $g_{0} \in L$ such that $x_{0}=g_{0} \Lambda$. Suppose that Theorem 3.1 fails to hold for some $\epsilon>0$. Then for any $R>0$ and any compact set $\mathcal{F}$ and infinitely many $i \in \mathbb{N}$, the condition (II) of Proposition 3.3 fails to hold for $J=I$, $h_{1}=a_{\boldsymbol{\tau}_{i}}$, and $h_{2}=g_{0}$; and hence the condition (I) holds.

Now take any sequence $R_{k} \stackrel{k \rightarrow \infty}{\longrightarrow} 0$ of positive reals. Then after passing to subsequences, there exists $W \in \mathscr{H}$, and for each $i$ there exists $\gamma_{i} \in \Lambda$ such that

$$
\sup _{s \in I}\left\|a_{\boldsymbol{\tau}_{i}} u(\varphi(s)) g_{0} \gamma_{i} p_{W}\right\| \leq R_{i} \stackrel{i \rightarrow \infty}{\longrightarrow} 0 .
$$

By Proposition 3.2, there exists $r_{0}>0$ such that $\left\|g_{0} \gamma_{i} p_{W}\right\| \geq r_{0}$ for each $i$. We put $v_{i}=g_{0} \gamma_{i} p_{W} /\left\|g_{0} \gamma_{i} p_{W}\right\|$. Then

$$
\sup _{s \in I}\left\|a_{\boldsymbol{\tau}_{i}} u(\varphi(s)) v_{i}\right\| \leq R_{i} /\left\|g_{0} \gamma_{i} p_{W}\right\| \leq R_{i} / r_{0} \stackrel{i \rightarrow \infty}{\longrightarrow} 0 .
$$

After passing to a subsequence, $v_{i} \rightarrow v \in V$ and $\|v\|=1$. Now from (3.3), (3.4), and (3.7) we deduce that

$$
u(\varphi(s)) v \in V_{\mathscr{T}}^{-}, \quad \forall s \in I .
$$

Since $\{\varphi(s): s \in I\}$ is not contained in a proper affine subspace of $\mathbb{R}^{n-1}$, (3.8) implies the if condition of (3.5) in Lemma 3.4 but contradicts its implication.

From Theorem 3.1 we deduce the following:

Corollary 3.5. After passing to a subsequence, $\mu_{i} \rightarrow \mu$ as $i \rightarrow \infty$ in the space of probability measures on $L / \Lambda$ with respect to the weak-* topology; that is,

$$
\int_{L / \Lambda} f d \mu_{i} \stackrel{i \rightarrow \infty}{\longrightarrow} \int_{L / \Lambda} f d \mu, \quad \forall f \in \mathrm{C}_{\mathrm{c}}(L / \Lambda) .
$$

\section{Dynamics of the intertwined linear actions of Various $\operatorname{SL}(m, \mathbb{R})$ 's CONTAINED IN $G$}

In this section we will give proofs of the new technical results of this article, including the one used above. We will also derive their further consequences, which will be crucially needed for applying the linearization techniques in combination with Ratner's theorem, in order to describe the limit measure $\mu$ in later sections.

4.1. Layered presentation for the infinite sequence $\mathscr{T}$. Let $\mathscr{T}=\left(\boldsymbol{\tau}_{i}\right)_{i \in \mathbb{N}}$ be an unbounded sequence as in $\$ 1.1$, that is, $\boldsymbol{\tau}_{i}=\left(\tau_{i, 1}, \ldots, \tau_{i, n-1}\right) \in \mathbb{R}^{n-1}$ such that $\tau_{i, 1} \geq \tau_{i, 2} \geq \cdots \geq \tau_{i, n-1} \geq 0$. By passing to a subsequence, we may further assume that there exist $k \in\{1, \ldots, n-1\}$ and integers $n-1 \geq m_{1}>m_{2}>\cdots>m_{k} \geq 1$ such that the following hold:

$$
\begin{array}{rlrl}
\lim _{i \rightarrow \infty} \tau_{i, r}<\infty & & \left(m_{1}<r \leq n-1\right), \\
\lim _{i \rightarrow \infty} \tau_{i, m_{1}} & =\infty, & & \\
\lim _{i \rightarrow \infty}\left(\tau_{i, m_{\ell+1}}-\tau_{i, m_{\ell}}\right) & =\infty & & (1 \leq \ell \leq k-1), \\
\lim _{i \rightarrow \infty}\left(\tau_{i, r}-\tau_{i, m_{\ell}}\right) & <\infty & & \left(m_{\ell+1}<r \leq m_{\ell}, 1 \leq \ell \leq k\right),
\end{array}
$$


where $m_{k+1}=0$. Define

$$
\begin{aligned}
\bar{\tau}_{i, r} & =0 \quad\left(m_{1}<r \leq n-1\right), \\
\bar{\tau}_{i, r} & =\tau_{i, m_{\ell}} \quad\left(m_{\ell+1}<r \leq m_{\ell}, 1 \leq \ell \leq k\right), \\
\bar{\tau}_{i} & =\left(\bar{\tau}_{i, 1}, \ldots, \bar{\tau}_{i, n-1}\right) .
\end{aligned}
$$

Then $\lim _{i \rightarrow \infty}\left(\boldsymbol{\tau}_{i}-\overline{\boldsymbol{\tau}}_{i}\right)$ exists in $\mathbb{R}^{n-1}$. Define

$$
\begin{aligned}
t_{i, 1} & =\bar{\tau}_{i, m_{1}}, \\
t_{i, \ell} & =\bar{\tau}_{i, m_{\ell}}-\bar{\tau}_{i, m_{\ell-1}} \quad(2 \leq \ell \leq k), \\
\boldsymbol{t}_{i} & =\left(t_{i, 1}, \ldots, t_{i, k}\right) \in\left(\mathbb{R}_{\geq 0}\right)^{k} .
\end{aligned}
$$

Thus we obtain a sequence $\mathcal{T}=\left(\boldsymbol{t}_{i}\right)_{i \in \mathbb{N}}$ associated to the given sequence $\mathscr{T}$ as above. Now $t_{i, \ell} \stackrel{i \rightarrow \infty}{\longrightarrow} \infty(1 \leq \ell \leq k)$.

4.2. Notation and set up. We are given natural numbers $n, k<n$, and $n-1 \geq$ $m_{1}>\cdots>m_{k} \geq 1$. Let $E_{i, j}$ denote the $n \times n$-matrix with 1 in the $(i, j)$-th coordinate and 0 in the rest. For $1 \leq \ell \leq k$, we define

$$
\mathcal{A}_{\ell}=m_{\ell} E_{1,1}-\sum_{j=2}^{m_{\ell}+1} E_{j, j}
$$

Given $1 \leq \ell \leq k$, let $\mathcal{T}=\left(\boldsymbol{t}_{i}\right)_{i=1}^{\infty} \subset\left(\mathbb{R}_{\geq 0}\right)^{\ell}$ be a sequence such that each coordinate of $\boldsymbol{t}_{i}$ tends to infinity as $i \rightarrow \infty$. For $\boldsymbol{t}=\left(t_{1}, \ldots, t_{\ell}\right) \in \mathbb{R}^{\ell}$, define

$$
\mathcal{A}(\boldsymbol{t}):=t_{1} \mathcal{A}_{1}+\cdots+t_{\ell} \mathcal{A}_{\ell} .
$$

Note that for the sequences $\mathscr{T}$ and $\mathcal{T}$ as described in 4.1 , we have

$$
a_{\overline{\boldsymbol{\tau}}_{i}}=\exp \left(\mathcal{A}\left(\boldsymbol{t}_{i}\right)\right), \quad \forall i \in \mathbb{N} .
$$

Let $V$ be a finite-dimensional linear representation of $\mathrm{SL}(n, \mathbb{R})$.

For $\boldsymbol{\mu}=\left(\mu_{1}, \ldots, \mu_{\ell}\right) \in \mathbb{R}^{\ell}$, we define

$$
V_{\boldsymbol{\mu}}=\left\{v \in V: \mathcal{A}_{i} v=\mu_{i} v \text { for } 1 \leq i \leq \ell\right\} .
$$

Thus if $v \in V_{\boldsymbol{\mu}}$, then $\mathcal{A}(\boldsymbol{t}) v=(\boldsymbol{\mu} \cdot \boldsymbol{t}) v$. The set $\Delta_{\ell}:=\left\{\boldsymbol{\nu} \in \mathbb{R}^{\ell}: V_{\boldsymbol{\nu}} \neq 0\right\}$ is finite, and

$$
V=\bigoplus_{\nu \in \Delta_{\ell}} V_{\nu}
$$

Let $\pi_{\boldsymbol{\mu}}^{\mathcal{T}}: V \rightarrow V_{\boldsymbol{\mu}}$ denote the corresponding projection. We put

$$
\Delta:=\Delta_{k} .
$$


We define

$$
\begin{aligned}
V^{-}(\mathcal{T})= & \left\{v \in V: \exp \left(\mathcal{A}\left(\boldsymbol{t}_{i}\right)\right) v \stackrel{i \rightarrow \infty}{\longrightarrow} 0\right\} \\
= & \sum_{\left\{\boldsymbol{\mu} \in \Delta_{\ell}: \lim _{i \rightarrow \infty} \boldsymbol{\mu} \cdot \boldsymbol{t}_{i}=-\infty\right\}} V_{\boldsymbol{\mu}}, \\
V^{+}(\mathcal{T})= & \left\{v \in V: \exp \left(-\mathcal{A}\left(\boldsymbol{t}_{i}\right)\right) v \stackrel{i \rightarrow \infty}{\longrightarrow} 0\right\} \\
= & \sum_{\left\{\boldsymbol{\mu} \in \Delta_{\ell}: \lim _{i \rightarrow \infty} \boldsymbol{\mu} \cdot \boldsymbol{t}_{i}=\infty\right\}} V_{\boldsymbol{\mu}}, \\
V^{0}(\mathcal{T})= & \left\{v \in V: \quad \begin{array}{l}
\text { both } \left.\exp \left(-\mathcal{A}\left(\boldsymbol{t}_{i}\right)\right) v \text { and } \exp \left(\mathcal{A}\left(\boldsymbol{t}_{i}\right)\right) v\right\} \\
=
\end{array} \sum_{\left\{\boldsymbol{\mu} \in \Delta_{\ell}:\left|\lim _{i \rightarrow \infty} \boldsymbol{\mu} \cdot \boldsymbol{t}_{i}\right|<\infty\right\}} V_{\boldsymbol{\mu}} .\right.
\end{aligned}
$$

Then after passing to a subsequence of $\mathcal{T}$, we have

$$
V=V^{+}(\mathcal{T}) \oplus V^{0}(\mathcal{T}) \oplus V^{-}(\mathcal{T}) .
$$

Let $\pi_{0}^{\mathcal{T}}: V \rightarrow V^{0}(\mathcal{T})$ denote the corresponding projection.

In view of (4.3) we have the following:

Lemma 4.1. Let $\mathscr{T}$ and $\mathcal{T}$ be as defined in 4.1 . Let $V_{\mathscr{T}}^{-}, V_{\mathscr{T}}^{+}$, and $V_{\mathscr{T}}^{0}$ be as defined in (3.3). Then

$$
V_{\mathscr{T}}^{-}=V^{+}(\mathcal{T}), \quad V_{\mathscr{T}}^{0}=V^{0}(\mathcal{T}), \quad \text { and } \quad V_{\mathscr{T}}^{+}=V^{0}(\mathcal{T}) .
$$

In particular, the corresponding projections $\pi_{0}^{\mathscr{T}}=\pi_{0}^{\mathcal{T}}$.

4.3. Main result. In the following discussion, let $\mathcal{T}=\left(\boldsymbol{t}_{i}\right)_{i=1}^{\infty} \subset\left(\mathbb{R}_{\geq 0}\right)^{k}$, where $k \geq 2$ is a sequence such that (each coordinate of $\boldsymbol{t}_{i} \stackrel{i \rightarrow \infty}{\longrightarrow} \infty$ and $V=V^{0}(\mathcal{T}) \oplus$ $V^{+}(\mathcal{T}) \oplus V^{-}(\mathcal{T})$. For $\boldsymbol{t}=\left(t_{1}, \ldots, t_{k}\right) \in \mathbb{R}^{k}$, let $\boldsymbol{t}^{\prime}=\left(t_{1}, \ldots, t_{k-1}\right) \in \mathbb{R}^{k-1}$. Given $\mathcal{T}$ as above, let

$$
\mathcal{T}^{\prime}=\left(\boldsymbol{t}_{i}^{\prime}\right)_{i=1}^{\infty} \subset\left(\mathbb{R}_{\geq 0}\right)^{k-1} .
$$

Proposition 4.2 (Basic Lemma II). Let $\mathcal{B}$ be an affine basis of $\mathbb{R}^{n-1}$; that is, $\{f-e: f \in \mathcal{B}\}$ is a basis of $\mathbb{R}^{n-1}$ for any $e \in \mathcal{B}$. Suppose that $v \in V$ is such that

$$
u(e) v \in V^{0}(\mathcal{T})+V^{-}(\mathcal{T}), \quad \forall e \in \mathcal{B} .
$$

Then for any $e \in \mathcal{B}$,

$$
u(e) v \in V^{0}\left(\mathcal{T}^{\prime}\right)+V^{-}\left(\mathcal{T}^{\prime}\right) .
$$

Moreover for any $e \in \mathcal{B}$,

$$
\text { if } \pi_{\mathbf{0}^{\prime}}^{\mathcal{T}^{\prime}}(u(e) v) \neq 0, \text { then } \pi_{\mathbf{0}}^{\mathcal{T}}(u(e) v) \neq 0,
$$

where $\mathbf{0}$ and $\mathbf{0}^{\prime}$ are the zero vectors of $\mathbb{R}^{k}$ and $\mathbb{R}^{k-1}$, respectively.

Remark 4.3. Our goal is to show that if condition (4.10) holds, then $\pi_{0}^{\mathcal{T}}(u(e) v) \neq 0$ for all $e \in \mathcal{B}$. To prove this, we apply conclusion (4.11) of Proposition 4.2 repeatedly and reduce the coordinates in $\mathcal{T}$ each time, till we are left with a sequence, say $\mathcal{T}(1)$, corresponding to only the first coordinates of elements of $\mathcal{T}$. Thus for all $e \in \mathcal{B}$, (4.11) is valid for $\mathcal{T}(1)$ in place of $\mathcal{T}^{\prime}$. Then we use the 'Basic Lemma' of 20] to conclude that $\pi_{0}^{\mathcal{T}(1)}(u(e) v) \neq 0$ for all $e \in \mathcal{B}$. But $V^{0}(\mathcal{T}(1))=V_{\mathbf{0}(1)}$, where $\mathbf{0}(1)=(0)$. Hence $\pi_{\mathbf{0}(1)}(u(e) v) \neq 0$. At that stage we use conclusion (4.12) 
repeatedly to increase one coordinate each time and finally get $\pi_{\mathbf{0}}^{\mathcal{T}}(u(e) v) \neq 0$ (see Corollary 4.10). Since $V_{\mathbf{0}} \subset V^{0}(\mathcal{T})$, we get $\pi_{0}^{\mathcal{T}}(u(e) v) \neq 0$.

The rest of $\$ 4.3$ is devoted to the proof of Proposition 4.2 .

4.3.1. Notation. Let $\mathfrak{c}=\operatorname{span}\left\{\mathcal{A}_{1}, \ldots, \mathcal{A}_{k}\right\}$. Let $\mathfrak{h}$ denote the Lie algebra generated by elements

$$
E_{1,1}, E_{1, j}, E_{j, 1}, E_{j, j}, \quad \text { where } 2 \leq j \leq m_{k}+1 .
$$

Then $\mathfrak{h}$ is naturally isomorphic to $\mathfrak{s l}\left(m_{k}+1, \mathbb{R}\right)$. Since $[\mathfrak{c}, \mathfrak{h}]=\mathfrak{h}$, we have that $\mathfrak{c}+\mathfrak{h}$ is a reductive subalgebra isomorphic to $\mathfrak{z}_{\mathfrak{c}}(\mathfrak{h}) \oplus \mathfrak{h}$, where $\mathfrak{z}_{\mathfrak{c}}(\mathfrak{h})$ denotes the centralizer of $\mathfrak{h}$ in $\mathfrak{c}$.

We define a preorder $\preceq$ on $\Delta$ by

$$
\boldsymbol{\mu} \preceq \boldsymbol{\nu} \Longleftrightarrow(\boldsymbol{\mu} \cdot \boldsymbol{t} \leq \boldsymbol{\nu} \cdot \boldsymbol{t} \text { for all } \boldsymbol{t} \in \mathcal{T}) .
$$

By passing to a subsequence of $\mathcal{T}$, we may assume that $\preceq$ is a total preorder. Note that $(\boldsymbol{\mu} \preceq \boldsymbol{\nu}$ and $\boldsymbol{\nu} \preceq \boldsymbol{\mu})$ does not imply $\boldsymbol{\mu}=\boldsymbol{\nu}$.

For $\boldsymbol{\mu}=\left(\mu_{1}, \ldots, \mu_{k}\right) \in \mathbb{R}^{k}$ we define $\boldsymbol{\mu}^{\prime}=\left(\mu_{1}, \ldots, \mu_{k-1}\right) \in \mathbb{R}^{k-1}$.

Lemma 4.4 (Positivity Lemma). Let $W$ be an irreducible $(\mathfrak{c}+\mathfrak{h})$-submodule of $V$. Let

$$
\Delta(W):=\left\{\boldsymbol{\mu} \in \Delta: \pi_{\boldsymbol{\mu}}^{\mathcal{T}}(W) \neq 0\right\} .
$$

Then for any $\boldsymbol{\mu}, \boldsymbol{\nu} \in \Delta(W)$ and $\boldsymbol{t} \in\left(\mathbb{R}_{>0}\right)^{k}$, where $k \geq 2$, we have

$$
\boldsymbol{\mu} \cdot \boldsymbol{t} \geq \boldsymbol{\nu} \cdot \boldsymbol{t} \Longleftrightarrow \mu_{k} \geq \nu_{k} \Longleftrightarrow \boldsymbol{\mu}^{\prime} \cdot \boldsymbol{t}^{\prime} \geq \boldsymbol{\nu}^{\prime} \cdot \boldsymbol{t}^{\prime},
$$

where $\boldsymbol{\mu}=\left(\mu_{1}, \ldots, \mu_{k}\right) \in \mathbb{R}^{k}$ and $\boldsymbol{\nu}=\left(\nu_{1}, \ldots, \nu_{k}\right) \in \mathbb{R}^{k}$.

Proof. In view of the expression $\mathfrak{c}+\mathfrak{h}=\mathfrak{z}_{\mathfrak{c}}(\mathfrak{h})+\mathfrak{h}$, for any $\boldsymbol{t}=\left(t_{1}, \ldots, t_{k}\right) \in \mathbb{R}^{k}$,

$$
\begin{aligned}
\mathcal{A}(\boldsymbol{t}) & =z\left(\boldsymbol{t}^{\prime}\right)+\left(f\left(\boldsymbol{t}^{\prime}\right)+t_{k}\right) \mathcal{A}_{k}, \\
\mathcal{A}\left(\boldsymbol{t}^{\prime}\right) & =z\left(\boldsymbol{t}^{\prime}\right)+f\left(\boldsymbol{t}^{\prime}\right) \mathcal{A}_{k},
\end{aligned}
$$

where $z\left(\boldsymbol{t}^{\prime}\right) \in \mathfrak{z}_{\mathfrak{c}}(\mathfrak{h})$ and

$$
f\left(\boldsymbol{t}^{\prime}\right)=\sum_{\ell=1}^{k-1} \frac{m_{\ell}+1}{m_{k}+1} t_{\ell}
$$

Here we observe that since $k \geq 2$,

$$
\boldsymbol{t}^{\prime} \in\left(\mathbb{R}_{>0}\right)^{k-1} \Longrightarrow f\left(\boldsymbol{t}^{\prime}\right)>0 .
$$

Since the action of $\mathfrak{c}$ is via commuting $\mathbb{R}$-diagonalizable elements and $W$ is an irreducible $\mathfrak{z}_{\mathfrak{c}}(\mathfrak{h})+\mathfrak{h}$-module, the center $\mathfrak{z}_{\mathfrak{c}}(\mathfrak{h})$ of $\mathfrak{c}+\mathfrak{h}$ acts on $W$ by scalars. Therefore for each $\boldsymbol{t} \in\left(\mathbb{R}_{>0}\right)^{k}$, there exists a constant $c\left(\boldsymbol{t}^{\prime}\right) \in \mathbb{R}$ such that

$$
z\left(\boldsymbol{t}^{\prime}\right) w=c\left(\boldsymbol{t}^{\prime}\right) w, \quad \forall w \in W .
$$

For any $\boldsymbol{\mu} \in \Delta(W)$, there exists $0 \neq w \in \pi_{\boldsymbol{\mu}}^{\mathcal{T}}(W) \subset W$. Now

$$
\begin{aligned}
(\boldsymbol{\mu} \cdot \boldsymbol{t}) w=\mathcal{A}(\boldsymbol{t}) w & =z\left(\boldsymbol{t}^{\prime}\right) w+\left(f\left(\boldsymbol{t}^{\prime}\right)+t_{k}\right) \mathcal{A}_{k} w \\
& =\left(c\left(\boldsymbol{t}^{\prime}\right)+\left(f\left(\boldsymbol{t}^{\prime}\right)+t_{k}\right) \mu_{k}\right) w,
\end{aligned}
$$

where $\boldsymbol{\mu} \cdot \boldsymbol{t}=\mu_{1} t_{1}+\cdots+\mu_{k} t_{k}$. Similarly,

$$
\left(\boldsymbol{\mu}^{\prime} \cdot \boldsymbol{t}^{\prime}\right) w=\mathcal{A}\left(\boldsymbol{t}^{\prime}\right) w=\left(c\left(\boldsymbol{t}^{\prime}\right)+f\left(\boldsymbol{t}^{\prime}\right) \mu_{k}\right) w .
$$


Therefore, since $w \neq 0$,

$$
\begin{aligned}
\boldsymbol{\mu} \cdot \boldsymbol{t} & =c\left(\boldsymbol{t}^{\prime}\right)+\left(f\left(\boldsymbol{t}^{\prime}\right)+t_{k}\right) \mu_{k}, \\
\boldsymbol{\mu}^{\prime} \cdot \boldsymbol{t}^{\prime} & =c\left(\boldsymbol{t}^{\prime}\right)+f\left(\boldsymbol{t}^{\prime}\right) \mu_{k} .
\end{aligned}
$$

Therefore, since $f\left(\boldsymbol{t}^{\prime}\right)>0$, for any $\boldsymbol{\mu}, \boldsymbol{\nu} \in \Delta(W)$,

$$
\boldsymbol{\mu} \cdot \boldsymbol{t} \geq \boldsymbol{\nu} \cdot \boldsymbol{t} \Longleftrightarrow \mu_{k} \geq \nu_{k} \Longleftrightarrow \boldsymbol{\mu}^{\prime} \cdot \boldsymbol{t}^{\prime} \geq \boldsymbol{\nu}^{\prime} \cdot \boldsymbol{t}^{\prime} .
$$

Notation. We express $\mathbb{R}^{n-1}=\mathbb{R}^{m_{k}} \oplus \mathbb{R}^{n-1-m_{k}}$ and let $q: \mathbb{R}^{n-1} \rightarrow \mathbb{R}^{m_{k}}$ and $q_{\perp}: \mathbb{R}^{n-1} \rightarrow \mathbb{R}^{n-1-m_{k}}$ denote the associated projections. Let

$$
\begin{aligned}
\mathfrak{W} & =\left\{\omega \in \mathrm{N}_{\mathrm{G}}\left(u\left(\mathbb{R}^{n-1}\right)\right): \exp \left(t \mathcal{A}_{k}\right) \omega \exp \left(-t \mathcal{A}_{k}\right) \stackrel{t \rightarrow \infty}{\rightarrow} e\right\} \\
& =\left\{\omega(w):=\left[\begin{array}{ccc}
1 & 0 & 0 \\
& I_{m_{k}} & w \\
& & I_{n-1-m_{k}}
\end{array}\right]: w \in M\left(m_{k} \times\left(n-1-m_{k}\right), \mathbb{R}\right)\right\} .
\end{aligned}
$$

Lemma 4.5. Let $\mathcal{E} \subset \mathbb{R}^{n-1}$ be such that $q(\mathcal{E})$ is a basis of $\mathbb{R}^{m_{k}}$. Then there exists $\omega \in \mathfrak{W}$ such that

$$
\omega u(e) \omega^{-1}=u(q(e)), \quad \forall e \in \mathcal{E} .
$$

Proof. We fix the standard basis of $\mathbb{R}^{n-1-m_{k}}$ and consider the basis $q(\mathcal{E})$ of $\mathbb{R}^{m_{k}}$. Then there exists a unique

$$
w \in \operatorname{End}\left(\mathbb{R}^{m_{k}}, \mathbb{R}^{n-1-m_{k}}\right) \cong M\left(m_{k} \times\left(n-1-m_{k}\right), \mathbb{R}\right)
$$

such that $w(q(e))=q_{\perp}(e)$ for all $e \in \mathcal{E}$. Then $\omega(w) \in \mathfrak{W}$ satisfies (4.29).

Lemma 4.6. For any $\omega \in \mathfrak{W}$ the following statements hold:

$$
\begin{aligned}
\omega\left(V^{0}(\mathcal{T})+V^{-}(\mathcal{T})\right) & \subset V^{0}(\mathcal{T})+V^{-}(\mathcal{T}), \\
x \in V^{0}(\mathcal{T})+V^{-}(\mathcal{T}) & \Rightarrow \pi_{0}^{\mathcal{T}}(x)=\pi_{0}^{\mathcal{T}}(\omega x), \\
\omega\left(V^{0}\left(\mathcal{T}^{\prime}\right)+V^{-}\left(\mathcal{T}^{\prime}\right)\right) & \subset V^{0}\left(\mathcal{T}^{\prime}\right)+V^{-}\left(\mathcal{T}^{\prime}\right), \\
x \in V^{0}\left(\mathcal{T}^{\prime}\right)+V^{-}\left(\mathcal{T}^{\prime}\right), \pi_{\mathbf{0}^{\prime}}^{\mathcal{T}^{\prime}}(x) \neq 0 & \Rightarrow \pi_{\mathbf{0}^{\prime}}^{\mathcal{T}^{\prime}}(\omega x) \neq 0 .
\end{aligned}
$$

Proof. Let $\mathfrak{w}$ denote the Lie subalgebra of $\mathfrak{g}$ associated to $\mathfrak{W}$. Then $\mathfrak{w}$ is contained in the sum of strictly negative eigenspaces of $\operatorname{ad}(\mathcal{A}(\mathcal{T}))$ acting on $\mathfrak{g}$. Therefore (4.31) and (4.32) hold.

Similarly (4.33) and (4.34) hold, because $\mathfrak{w}$ is contained in the sum of zero eigenspaces and strictly negative eigenspaces of $\operatorname{ad}\left(\mathcal{A}_{\ell}\right)$ acting on $\mathfrak{g}$ for all $1 \leq \ell \leq$ $k-1$.

One of the crucial ingredients in the proof of Proposition 4.2 is following 'Basic Lemma I' [20, Proposition 4.2].

Proposition 4.7. Let $m \geq 1$ and $\mathcal{A}=\operatorname{diag}(m,-1, \ldots,-1) \in \mathfrak{s l}(m+1, \mathbb{R})$. For any $\boldsymbol{x} \in \mathbb{R}^{m}$, let $u(\boldsymbol{x})=\left(\begin{array}{cc}1 & \boldsymbol{x} \\ 0 & I_{m}\end{array}\right) \in \mathfrak{s l}(m+1, \mathbb{R})$. Let $W$ be a finite-dimensional representation of $\mathrm{SL}(m+1, \mathbb{R})$. Let $W^{-}$(respectively $\left.W^{+}\right)$be the sum of strictly negative (respectively positive) eigenspaces of $\mathcal{A}$ and let $W^{0}$ be the null space of $\mathcal{A}$. Let $\pi_{0}: W \rightarrow W^{0}$ denote the projection parallel to $W^{-} \oplus W^{+}$. Let $\overline{\mathcal{B}}_{1}$ be an affine basis of $\mathbb{R}^{m}$ and let $w \in W$. Suppose that

$$
u(e) w \in W^{0}+W^{-}, \quad \forall e \in \overline{\mathcal{B}}_{1} .
$$


Then

$$
\pi_{0}(u(e) w) \neq 0, \quad \forall e \in \overline{\mathcal{B}}_{1} .
$$

Corollary 4.8. In Proposition 4.7, suppose further that $\pi_{0}\left(u\left(e_{0}\right) w\right)$ is fixed by $\mathrm{SL}(m+1, \mathbb{R})$ for some $e_{0} \in \overline{\mathcal{B}}_{1}$. Then $w$ is fixed by $\mathrm{SL}(m+1, \mathbb{R})$.

Proof. Let $w_{0}=u\left(e_{0}\right) w-\pi_{0}\left(u\left(e_{0}\right) w\right)$. Put $\overline{\mathcal{B}}_{2}=\left\{e-e_{0}: e \in \overline{\mathcal{B}}_{1}\right\}$. Then $u\left(e^{\prime}\right) w_{0} \in$ $V^{0}+V^{-}$for all $e^{\prime} \in \overline{\mathcal{B}}_{2}$. Since $\overline{\mathcal{B}}_{2}$ is an affine basis of $\mathbb{R}^{m}$, by Proposition 4.7, if $w_{0} \neq 0$, then $\pi_{0}\left(w_{0}\right) \neq 0$, which contradicts the choice of $w_{0}$. Therefore $w_{0}=0$. Hence $u\left(e_{0}\right) w=\pi_{0}\left(u\left(e_{0}\right) w\right)$ is fixed by $\operatorname{SL}(m+1, \mathbb{R})$. In turn, $w$ is fixed by $\mathrm{SL}(m+1, \mathbb{R})$.

Proof of Proposition 4.2. Let $e_{0} \in \mathcal{B}$. We want to prove (4.11) and (4.12) for $e_{0}$ in place of $e$. By replacing $v$ by $u\left(e_{0}\right) v$ and replacing every element $e \in \mathcal{B}$ by $e-e_{0}$, without loss of generality we may assume that $e_{0}=0$. Let $\mathcal{B}_{1} \subset \mathcal{B}$ containing 0 such that $q\left(\mathcal{B}_{1}\right) \backslash\{0\}$ is a basis of $\mathbb{R}^{m_{k}}$. By Lemma 4.5 there exists $\omega \in \mathfrak{W}$ such that

$$
\omega u(e) \omega^{-1}=u(q(e)) \quad \forall e \in \mathcal{B}_{1} .
$$

We put $v_{0}:=\omega v$. Then by (4.10), (4.31), and (4.37), we have

$$
u(q(e)) v_{0}=\omega(u(e) v) \subset V^{0}(\mathcal{T})+V^{-}(\mathcal{T}), \quad \forall e \in \mathcal{B}_{1} .
$$

By (4.34), if $\pi_{\mathbf{0}^{\prime}}^{\mathcal{T}^{\prime}}(v) \neq 0$, then $\pi_{\mathbf{0}^{\prime}}^{\mathcal{T}^{\prime}}\left(v_{0}\right) \neq 0$. By (4.32), if $\pi_{\mathbf{0}}^{\mathcal{T}}\left(v_{0}\right) \neq 0$, then $\pi_{\mathbf{0}}^{\mathcal{T}}(v) \neq$ 0 . Therefore by (4.33), in order to prove (4.11) and (4.12), it is enough to show that

$$
\begin{gathered}
v_{0}=\omega v \in V^{0}\left(\mathcal{T}^{\prime}\right)+V^{-}\left(\mathcal{T}^{\prime}\right) \text { and } \\
\text { if } \pi_{\mathbf{0}}^{\mathcal{T}^{\prime}}\left(v_{0}\right) \neq 0 \text {, then } \pi_{\mathbf{0}}^{\mathcal{T}}\left(v_{0}\right) \neq 0 .
\end{gathered}
$$

We decompose $V$ into irreducible $(\mathfrak{c}+\mathfrak{h})$-submodules as

$$
V=W_{1} \oplus \cdots \oplus W_{s} .
$$

For each $1 \leq j \leq s$, let $P_{j}: V \rightarrow W_{j}$ denote the associated projection, which is $(\mathfrak{c}+\mathfrak{h}$ )-equivariant. To show the validity of (4.39) and (4.40), it is enough to prove that for every $1 \leq j \leq s$,

$$
\begin{gathered}
P_{j}\left(v_{0}\right) \in V^{0}\left(\mathcal{T}^{\prime}\right)+V^{-}\left(\mathcal{T}^{\prime}\right) \quad \text { and } \\
\mathcal{A}_{k} \cdot P_{j}\left(\pi_{\mathbf{0}^{\prime}}^{\mathcal{T}^{\prime}}\left(v_{0}\right)\right)=0 .
\end{gathered}
$$

Now henceforth we will fix $j$ as above and put $W=W_{j}$ and $P=P_{j}$.

Without loss of generality we may suppose that $P\left(v_{0}\right) \neq 0$. Let

$$
\Delta\left(W, \mathcal{B}_{1}\right)=\left\{\boldsymbol{\nu} \in \Delta(W): \pi_{\boldsymbol{\nu}}\left(P\left(u(q(e)) v_{0}\right)\right) \neq 0 \quad \text { for some } e \in \mathcal{B}_{1}\right\} .
$$

We now recall that by (4.38) we have

$$
\boldsymbol{\nu} \cdot \boldsymbol{t} \leq 0, \quad \forall \boldsymbol{\nu} \in \Delta\left(W, \mathcal{B}_{1}\right), \forall \boldsymbol{t} \in \mathcal{T} .
$$

Let $\boldsymbol{\mu}$ be the maximal element of $\Delta\left(W, \mathcal{B}_{1}\right) \neq \emptyset$ with respect to the total preorder defined on $\Delta$; that is,

$$
\boldsymbol{\nu} \cdot \boldsymbol{t} \leq \boldsymbol{\mu} \cdot \boldsymbol{t}, \quad \forall \boldsymbol{t} \in \mathcal{T}, \forall \boldsymbol{\nu} \in \Delta\left(W, \mathcal{B}_{1}\right) .
$$


Therefore by Lemma 4.4, for all $\boldsymbol{\nu} \in \Delta\left(W, \mathcal{B}_{1}\right)$ we have

$$
\begin{aligned}
\nu_{k} & \leq \mu_{k} \quad \text { and } \\
\boldsymbol{\nu}^{\prime} \cdot \boldsymbol{t}^{\prime} & \leq \boldsymbol{\mu}^{\prime} \cdot \boldsymbol{t}, \quad \forall \boldsymbol{t} \in \mathcal{T} .
\end{aligned}
$$

For $\lambda \in \mathbb{R}$, let

$$
W_{\lambda}=\left\{w \in W: \mathcal{A}_{k} w=\lambda w\right\} .
$$

Then for any $\boldsymbol{\nu}=\left(\nu_{1}, \ldots, \nu_{k}\right) \in \Delta$,

$$
\pi_{\nu}\left(P\left(u(q(e)) v_{0}\right)\right) \in W_{\nu_{k}} .
$$

Hence by (4.44), (4.47), and (4.50) we conclude that

$$
u(q(e)) P\left(v_{0}\right)=P\left(u(q(e)) v_{0}\right) \in \sum_{\lambda \leq \mu_{k}} W_{\lambda}, \quad \forall e \in \mathcal{B}_{1} .
$$

Let $H$ be the Lie subgroup of $G$ associated to the Lie algebra $\mathfrak{h}$. Then $H$ is naturally isomorphic to $\mathrm{SL}\left(m_{k}+1, \mathbb{R}\right)$. We now apply Proposition 4.7 in the case of $m=m_{k}$ and $\mathcal{A}=\mathcal{A}_{k}, \overline{\mathcal{B}}_{1}=q\left(\mathcal{B}_{1}\right)$ and $w=P\left(v_{0}\right)$. Note that if $\mu_{k}<0$, then by (4.51) we have

$$
u(\bar{e}) w \in W^{-}, \quad \forall \bar{e} \in \overline{\mathcal{B}}_{1} .
$$

Therefore the condition (4.35) of Proposition 4.7 is satisfied but its conclusion (4.36) fails to hold. Thus we conclude that

$$
\mu_{k} \geq 0 \text {. }
$$

Therefore for any $\boldsymbol{t} \in \mathcal{T}$, by (4.45), we have

$$
\boldsymbol{\mu}^{\prime} \cdot \boldsymbol{t}^{\prime}=\boldsymbol{\mu} \cdot \boldsymbol{t}-\mu_{k} t_{k} \leq 0 .
$$

Then from (4.48) we conclude that

$$
\boldsymbol{\nu}^{\prime} \cdot \boldsymbol{t}^{\prime} \leq \boldsymbol{\mu}^{\prime} \cdot \boldsymbol{t}^{\prime} \leq 0, \quad \forall \boldsymbol{\nu} \in \Delta\left(W, \mathcal{B}_{1}\right), \forall \boldsymbol{t}^{\prime} \in \mathcal{T}^{\prime} .
$$

Now in view of (4.44) this implies (4.42).

Next in order to prove (4.43), suppose that $\boldsymbol{\nu} \in \Delta\left(W, \mathcal{B}_{1}\right)$ and $\boldsymbol{\nu}^{\prime}=\mathbf{0}^{\prime}$. We need to show that $\boldsymbol{\nu}=0$.

Let $i \in \mathbb{N}$. By (4.55),

$$
0=\boldsymbol{\nu}^{\prime} \cdot \boldsymbol{t}_{i}^{\prime} \leq \boldsymbol{\mu}^{\prime} \cdot \boldsymbol{t}_{i}^{\prime} \leq 0 .
$$

Therefore, by (4.53) and since $\boldsymbol{t}_{i, k}>0$,

$$
0 \geq \boldsymbol{\mu} \cdot \boldsymbol{t}_{i}=\boldsymbol{\mu}^{\prime} \cdot \boldsymbol{t}_{i}^{\prime}+\mu_{k} t_{i, k} \geq 0,
$$

and hence

$$
\mu_{k}=0 .
$$

Therefore by (4.26) and (4.56), applied first to $\boldsymbol{\nu}$ and then to $\boldsymbol{\mu}$, we get

$$
f\left(\boldsymbol{t}_{i}^{\prime}\right) \nu_{k}=\boldsymbol{\nu}^{\prime} \cdot \boldsymbol{t}_{i}^{\prime}-c\left(\boldsymbol{t}_{i}^{\prime}\right)=-c\left(\boldsymbol{t}_{i}^{\prime}\right)=f\left(\boldsymbol{t}^{\prime}\right) \mu_{k}-\boldsymbol{\mu}^{\prime} \cdot \boldsymbol{t}_{i}^{\prime}=0 .
$$

Since $f\left(\boldsymbol{t}_{i}^{\prime}\right)>0$, we conclude that $\boldsymbol{\nu}=0$. 
4.4. Consequences of the Basic Lemma II. For any $1 \leq \ell \leq k$ and $\boldsymbol{t}=$ $\left(t_{1}, \ldots, t_{k}\right) \in \mathbb{R}^{k}$ define $\boldsymbol{t}(\ell)=\left(t_{1}, \ldots, t_{\ell}\right)$ and $\mathcal{T}(\ell)=\left(\boldsymbol{t}_{i}(\ell)\right)_{i=1}^{\infty}$, where $\mathcal{T}=\left(\boldsymbol{t}_{i}\right)_{i=1}^{\infty}$ such that $\boldsymbol{t}_{i} \in\left(\mathbb{R}_{\geq 0}\right)^{k}$, each coordinate of $\boldsymbol{t}_{i}$ tends to infinity as $i \rightarrow \infty$. By passing to a subsequence, we will further assume that

$$
V=V^{-}(\mathcal{T}(\ell)) \oplus V^{0}(\mathcal{T}(\ell)) \oplus V^{-}(\mathcal{T}(\ell)) \quad(1 \leq \ell \leq k)
$$

By applying Proposition 4.2 repeatedly, we can decrease $k$ and obtain the following:

Proposition 4.9. Let $\mathcal{B}$ be an affine basis of $\mathbb{R}^{n-1}$ and let $v \in V$ be such that

$$
u(e) v \in V^{0}(\mathcal{T})+V^{-}(\mathcal{T}), \quad \forall e \in \mathcal{B} .
$$

Then for any $1 \leq \ell \leq k$ and any $e \in \mathcal{B}$,

$$
\begin{gathered}
u(e) v \in V^{0}(\mathcal{T}(\ell))+V^{-}(\mathcal{T}(\ell)) \text { and } \\
\text { if } \pi_{\mathbf{0}(\ell)}^{\mathcal{T}(\ell)}(u(e) v) \neq 0 \text {, then } \pi_{\mathbf{0}}^{\mathcal{T}}(u(e) v) \neq 0 .
\end{gathered}
$$

By specializing this result to the case of $\ell=1$, we deduce the following generalization of Proposition 4.7 .

Corollary 4.10. Let $\mathcal{B}$ be an affine basis of $\mathbb{R}^{n-1}$ and let $v \in V$ be such that

$$
u(e) v \in V^{0}(\mathcal{T})+V^{-}(\mathcal{T}), \quad \forall e \in \mathcal{B} .
$$

Then

$$
\pi_{\mathbf{0}}^{\mathcal{T}}(u(e) v) \neq 0, \quad \forall e \in \mathcal{B}
$$

Proof. We apply Proposition 4.9 for $\ell=1$. Then from (4.62) we get

$$
u(e) v \in V^{0}(\mathcal{T}(1))+V^{-}(\mathcal{T}(1)), \quad \forall e \in \mathcal{B} .
$$

Take any $e_{0} \in \mathcal{B}$ and choose $\mathcal{B}_{1} \subset \mathcal{B}$ containing $e_{0}$ such that $q\left(\mathcal{B}_{1}\right)$ is an affine basis of $\mathbb{R}^{m_{1}}$. Let $\mathfrak{W}$ be defined as in (4.28) associated to $\mathcal{A}_{1}$ in place of $\mathcal{A}_{k}$. Then by Lemma 4.5 there exists $\omega \in \mathfrak{W}$ such that $\omega u\left(e-e_{0}\right) \omega^{-1}=u\left(q\left(e-e_{0}\right)\right)$ for all $e \in \mathcal{B}_{1}$. Therefore

$$
u\left(q\left(e-e_{0}\right)\right)\left(\omega u\left(e_{0}\right) v\right) \in V^{0}(\mathcal{T}(1))+V^{-}(\mathcal{T}(1)), \quad \forall e \in \mathcal{B}_{1} .
$$

Therefore by Proposition 4.7 applied to $m=m_{1}, \mathcal{A}=\mathcal{A}_{1}, \overline{\mathcal{B}}_{1}=\left\{q\left(e-e_{0}\right): e \in \mathcal{B}_{1}\right\}$, and $w=\omega u\left(e_{0}\right) v$, we get

$$
\pi_{\mathbf{0}(1)}^{\mathcal{T}(1)}\left(\omega u\left(e_{0}\right) v\right) \neq 0 .
$$

Therefore in view of (4.32) of Lemma 4.6 for $\mathfrak{W}$ and $\mathcal{T}$ defined for the case of $k=1$, we get

$$
\pi_{\mathbf{0}(1)}^{\mathcal{T}(1)}\left(u\left(e_{0}\right) v\right)=\pi_{\mathbf{0}(1)}^{\mathcal{T}(1)}\left(\omega u\left(e_{0}\right) v\right) \neq 0 .
$$

Now from (4.63) of Proposition 4.9 we conclude that $\pi_{\mathbf{0}}^{\mathcal{T}}\left(u\left(e_{0}\right) v\right) \neq 0$.

Corollary 4.11. Let $\mathcal{T} \subset\left(\mathbb{R}_{\geq 0}\right)^{k}$ be as in $₫ 4.3$. Assume that $m_{1}=n-1$. Let $\varphi: I=[a, b] \rightarrow \mathbb{R}^{n-1}$ be a differentiable curve whose image is not contained in a proper affine subspace of $\mathbb{R}^{n-1}$. Let $v \in V$ be such that

$$
u(\varphi(s)) v \in V^{0}(\mathcal{T})+V^{-}(\mathcal{T}) .
$$

Then $v$ is $G$-fixed. 
Proof. Given any $s_{0} \in I$, there exists a set $\left\{s_{1}, \ldots, s_{n-1}\right\} \subset I$ such that $\mathcal{B}:=$ $\left\{\varphi\left(s_{i}\right): 0 \leq i \leq n-1\right\}$ is an affine basis of $\mathbb{R}^{n-1}$. Therefore by (4.62) of Proposition 4.9 applied to $\ell=1$ we get

$$
u\left(\varphi\left(s_{0}\right)\right) v \in V^{0}(\mathcal{T}(1))+V^{-}(\mathcal{T}(1)) .
$$

Therefore by [20, Corollary 4.6] $G$ fixes $v$.

We will now generalize the above result for all $1 \leq m_{1} \leq n-1$.

Proposition 4.12. Let $\mathcal{T} \subset\left(\mathbb{R}_{\geq 0}\right)^{k}$ be as 4 4.3. Let $\varphi: I=[a, b] \rightarrow \mathbb{R}^{n-1}$ be a differentiable curve which is not contained in a proper affine subspace of $\mathbb{R}^{n-1}$. Let $v \in V$ be such that

$$
u(\varphi(s)) v \in V^{0}(\mathcal{T})+V^{-}(\mathcal{T}), \quad \forall s \in I .
$$

Then $v$ is fixed by the subgroup $Q_{m_{1}+1}$.

Proof. Without loss of generality we may assume that $v \neq 0$. In view of Proposition 4.9

$$
u(\varphi(s)) v \in V^{0}(\mathcal{T}(1))+V^{-}(\mathcal{T}(1)), \quad \forall s \in I .
$$

At this stage we will take $k=1$ and replace $\mathcal{T}$ by $\mathcal{T}(1)$. Let $L=\mathbb{R}^{m_{1}}, L^{\perp}=$ $\mathbb{R}^{n-1-m_{1}}$, and let $q: \mathbb{R}^{n-1} \rightarrow L$ and $q_{\perp}: \mathbb{R}^{n-1} \rightarrow L^{\perp}$ be the projections associated to the decomposition $\mathbb{R}^{n-1}=L \oplus L^{\perp}$.

Take any $s_{0} \in I$ and put $e_{0}=\varphi\left(s_{0}\right)$. Due to the hypothesis on $\varphi$ there exists a finite set $\mathcal{E} \subset \varphi(I)$ containing $e_{0}$ such that $\left\{q(e)-q\left(e_{0}\right): e \in \mathcal{E}\right\}$ is not contained in a union of $\left(m_{1}+1\right)$ proper subspaces of $L$.

Let $\mathcal{B}_{1} \subset \mathcal{E}$ containing $e_{0}$ be such that the set $\left\{q(e)-q\left(e_{0}\right): e \in \mathcal{B}_{1} \backslash\left\{e_{0}\right\}\right\}$ is a basis of $L$. Let $\mathfrak{W}$ be defined as in (4.28) for $k=1$. Then there exists $\omega \in \mathfrak{W}$ such that $\omega u\left(e-e_{0}\right) \omega^{-1}=u\left(q\left(e-e_{0}\right)\right)$ for all $e \in \mathcal{B}_{1}$. We put $v_{0}=u\left(e_{0}\right) v$. Now by (4.73) and (4.31) for the case of $k=1$,

$$
\begin{aligned}
u\left(q\left(e-e_{0}\right)\right) \omega v_{0} & =\omega u\left(e-e_{0}\right) v_{0} \\
& =\omega u(e) v \in V^{0}\left(\mathcal{A}_{1}\right) \oplus V^{-}\left(\mathcal{A}_{1}\right) .
\end{aligned}
$$

Let $H \cong \mathrm{SL}\left(m_{1}+1, \mathbb{R}\right)$ be the Lie group associated to the Lie algebra $\mathfrak{h}$ as defined through (4.13) for $k=1$. Let $\mathcal{C}=\left\{q\left(e-e_{0}\right): e \in \mathcal{B}_{1} \backslash\left\{e_{0}\right\}\right\}$. Let $D_{\mathcal{C}}$ consist of those $g \in Z_{H}\left(\exp \left(\mathbb{R} \mathcal{A}_{1}\right)\right)$ such that for each $e^{\prime} \in \mathcal{C}$, we have $g u\left(e^{\prime}\right) g^{-1}=u\left(\lambda e^{\prime}\right)$ for some $\lambda>0$; cf. [20, eq. (4.48)]. Then by [20, Proposition 2.3], for all $e \in \mathcal{B}_{1}$,

$$
\pi_{0}^{\mathcal{A}_{1}}(\omega u(e) v) \neq 0 \quad \text { and } \quad D_{\mathcal{C}} \subset \operatorname{Stab}_{G}\left(\pi_{0}^{\mathcal{A}_{1}}(\omega u(e) v)\right) .
$$

By (4.32), applied to the case of $k=1, \pi_{0}^{\mathcal{A}_{1}}\left(\omega v_{0}\right)=\pi_{0}^{\mathcal{A}_{1}}\left(v_{0}\right)$, and hence

$$
\pi_{0}^{\mathcal{A}_{1}}\left(v_{0}\right) \neq 0 \quad \text { and } \quad D_{\mathcal{C}} \subset \operatorname{Stab}_{G}\left(\pi_{0}^{\mathcal{A}_{1}}\left(v_{0}\right)\right) .
$$

This equation holds for all choices of $\mathcal{B}_{1} \subset \mathcal{E}$ containing $e_{0}$ such that $q\left(\mathcal{B}_{1}\right)$ spans $L$; here it is important that (4.76) does not involve $\omega$. Therefore in view of the hypothesis on $\mathcal{E}$, by [20, Corollary 2.4] we can deduce that

$$
\begin{gathered}
\pi_{0}^{\mathcal{A}_{1}}\left(v_{0}\right) \neq 0 \quad \text { and } \\
Z_{H}\left(\exp \left(\mathbb{R} \mathcal{A}_{1}\right)\right) \subset \operatorname{Stab}_{G}\left(\pi_{0}^{\mathcal{A}_{1}}\left(v_{0}\right)\right) .
\end{gathered}
$$

Next we want to show that

$$
u(\lambda q(\dot{\varphi}(s))) \in \operatorname{Stab}_{G}\left(\pi_{0}^{\mathcal{A}_{1}}(u(\varphi(s) v))\right), \quad \forall s \in I \text { and } \lambda \in \mathbb{R} .
$$


To see this, put $a(t)=\exp \left(t \mathcal{A}_{1}\right)$ for all $t \in \mathbb{R}$. For $\xi \in \mathbb{R}^{n-1}$, we define $a(t) \cdot \xi$ by the relation $u(a(t) \cdot \xi)=a(t) u(\xi) a(t)^{-1}$. Then $a(t) \cdot q(\xi)=e^{m_{1} t} q(\xi), a(t) \cdot q_{\perp}(\xi)=$ $e^{\left(m_{1}-1\right) t} q_{\perp}(\xi)$, and hence

$$
e^{-m_{1} t} a(t) \cdot \xi \stackrel{t \rightarrow \infty}{\longrightarrow} q(\xi) .
$$

Take $\lambda \in \mathbb{R}$ and $t_{i} \stackrel{i \rightarrow \infty}{\longrightarrow} \infty$. Put $s_{i}=s+\lambda e^{-m_{1} t_{i}}$. Then

$$
\begin{aligned}
a\left(t_{i}\right) u\left(\varphi\left(s_{i}\right)\right) v & =\pi_{0}^{\mathcal{A}_{1}}\left(u\left(\varphi\left(s_{i}\right)\right)\right)+a\left(t_{i}\right) \pi_{-}^{\mathcal{A}_{1}}\left(u\left(\varphi\left(s_{i}\right)\right)\right) \\
& \stackrel{i \rightarrow \infty}{\longrightarrow} \pi_{0}^{\mathcal{A}_{1}}(u(\varphi(s))) .
\end{aligned}
$$

Also $a\left(t_{i}\right) u(\varphi(s)) v \stackrel{i \rightarrow \infty}{\longrightarrow} \pi_{0}^{\mathcal{A}_{1}}(u(\varphi(s)) v)$. Since

$$
\begin{aligned}
\varphi\left(s_{i}\right)-\varphi(s) & =\left(s_{i}-s\right) \dot{\varphi}(s)+O\left(\left(s_{i}-s\right)^{2}\right) \\
& =e^{-m_{1} t_{i}} \dot{\varphi}(s)+O\left(e^{-2 m_{1} t_{i}}\right),
\end{aligned}
$$

by (4.80),

$$
a_{i} \cdot\left(\varphi\left(s_{i}\right)-\varphi\left(s_{0}\right)\right) \stackrel{i \rightarrow \infty}{\longrightarrow} \lambda q(\dot{\varphi}(s))
$$

Therefore

$$
\begin{aligned}
a_{i} u\left(\varphi\left(s_{i}\right)\right) v & =a_{i} u\left(\varphi\left(s_{i}\right)-\varphi(s)\right) u(\varphi(s)) v \\
& =u\left(a_{i} \cdot\left(\varphi\left(s_{i}\right)-\varphi(s)\right)\right) a_{i} u(\varphi(s)) v \\
& \stackrel{i \rightarrow \infty}{\longrightarrow} u(\lambda q(\dot{\varphi}(s))) \pi_{0}^{\mathcal{A}_{1}}(u(\varphi(s)) v) .
\end{aligned}
$$

Thus (4.79) follows from (4.81) and (4.84).

Due to our hypothesis on $\varphi(s)$, we could choose $s_{0} \in I$ such that $q\left(\dot{\varphi}\left(s_{0}\right)\right) \neq 0$. Let $Q$ denote the subgroup of $H$ generated by $Z_{H}\left(\exp \left(\mathbb{R} \mathcal{A}_{1}\right)\right)$ and $u\left(\mathbb{R} q\left(\dot{\varphi}\left(s_{0}\right)\right)\right)$. It may be verified that $Q$ is a parabolic subgroup of $H$. By (4.77) and (4.79), $Q \subset \operatorname{Stab}_{G}\left(\pi_{0}^{\mathcal{A}_{1}}\right)$. Therefore we conclude that

$$
H \subset \operatorname{Stab}_{G}\left(\pi_{0}^{\mathcal{A}_{1}}\left(v_{0}\right)\right) .
$$

Therefore $H$ fixes $\pi_{0}^{\mathcal{A}_{1}}\left(\omega v_{0}\right)=\pi_{0}^{\mathcal{A}_{1}}\left(v_{0}\right)$. Now by (4.74) and Corollary 4.8 we conclude that

$$
H \subset \operatorname{Stab}_{G}\left(\omega v_{0}\right) .
$$

Let $\left\{e_{1}, \ldots, e_{m_{1}}\right\}$ denote the standard basis of $\mathbb{R}^{m_{1}}=L \subset \mathbb{R}^{n-1}$. Note that any $z \in Z_{H}\left(\mathbb{R} \mathcal{A}_{1}\right)$ acts on $e \in L$ via the relation $u(z \cdot e)=z u(e) z^{-1}$. This action of $Z_{H}\left(\mathbb{R} \mathcal{A}_{1}\right)$ surjects onto $\mathrm{GL}\left(m_{1}, \mathbb{R}\right)$. Therefore there exists $z \in \mathbb{Z}_{H}\left(\mathbb{R} \mathcal{A}_{1}\right)$ such that

$$
\left\{z \cdot q\left(e-e_{0}\right): e \in \mathcal{B}_{1}\right\}=\left\{0, e_{1}, \ldots, e_{m_{1}}\right\} .
$$

Since $\omega \in Q_{m_{1}+1}$ and

$$
z \omega\left(V^{0}\left(\mathcal{A}_{1}\right)+V^{-}\left(\mathcal{A}_{1}\right)\right)=V^{0}\left(\mathcal{A}_{1}\right)+V^{-}\left(\mathcal{A}_{1}\right),
$$

replacing $\varphi(s)$ by the curve $\varphi_{1}(s)$ such that

$$
z \omega u\left(\varphi(s)-e_{0}\right) \omega^{-1} z^{-1}=u\left(\varphi_{1}(s)\right), \quad \forall s \in I,
$$

and replacing $v$ by $z \omega v_{0}$ and $\mathcal{B}_{1}$ by $\left\{0, e_{1}, \ldots, e_{m_{1}}\right\}$, without loss of generality we may assume the following:

$$
H \cdot v=v, \quad \text { and } \quad u(\varphi(s)) v \in V^{0}\left(\mathcal{A}_{1}\right)+V^{-}\left(\mathcal{A}_{1}\right), \quad \forall s \in I .
$$


Let $q_{1}: \mathbb{R}^{n-1} \rightarrow \mathbb{R} e_{m_{1}+1}$ denote the coordinate projection. Put

$$
\mathcal{F}=\left\{\varphi(s): s \in I, q_{1}(\varphi(s)) \neq 0\right\} ; \text { then } L^{\perp}=\operatorname{span}\left(\left\{q_{\perp}(e): e \in \mathcal{F}\right\}\right) .
$$

Now take any $e \in \mathcal{F}$. Then there exists $z_{e} \in Z_{H}\left(\exp \left(\mathbb{R} \mathcal{A}_{1}\right)\right)$ such that

$$
z_{e} \cdot q(e)=e_{1} \quad \text { and } \quad z_{e} \cdot e_{i}=e_{i} \quad\left(2 \leq i \leq m_{k}\right) .
$$

Therefore, since elements of $H$ and $u\left(L^{\perp}\right)$ commute, we have

$$
z_{e} u(e) z_{e}{ }^{-1}=u\left(e_{1}+q_{\perp}(e)\right) .
$$

Write $q_{\perp}(e)=\left(x_{m_{1}+1}(e), \ldots, x_{n-1}(e)\right) \in L^{\perp} \cong \mathbb{R}^{n-1-m_{1}}$, and let

$$
\omega_{e}:=I_{n}+\sum_{j=m_{1}+1}^{n-1} x_{j}(e) E_{2,(1+j)} \in \mathfrak{W} .
$$

Then $\omega_{e} u\left(e_{1}+q_{\perp}(e)\right) \omega_{e}^{-1}=u\left(e_{1}\right)$ and $\omega_{e}$ commutes with $u\left(e_{i}\right)$ for $i \geq 2$. Therefore by (4.90), (4.92), and (4.93),

$$
\begin{aligned}
& V^{0}\left(\mathcal{A}_{1}\right)+V^{-}\left(\mathcal{A}_{1}\right) \ni \omega_{e} z_{e} u(e) v=u\left(e_{1}\right)\left(\omega_{e} v\right), \\
& V^{0}\left(\mathcal{A}_{1}\right)+V^{-}\left(\mathcal{A}_{1}\right) \ni \omega_{e} z_{e} u\left(e_{i}\right) v=u\left(e_{i}\right)\left(\omega_{e} v\right) \quad\left(2 \leq i \leq m_{k}\right) .
\end{aligned}
$$

Therefore, since $\pi_{0}^{\mathcal{A}}\left(\omega_{e} v\right)=\pi_{0}^{\mathcal{A}}(v)=v$ is fixed by $H$, by Corollary 4.8

$$
H \subset \operatorname{Stab}_{G}\left(\omega_{e} v\right) .
$$

Now $H \cup\left(\omega_{e} H \omega_{e}{ }^{-1}\right) \subset \operatorname{Stab}(v), \exp \left(\mathbb{R} \mathcal{A}_{1}\right) \subset H$, and $\exp \left(t \mathcal{A}_{1}\right) \omega_{e} \exp \left(-t A_{1}\right)$ converges to the identity element as $t \rightarrow \infty$. Therefore $\omega_{e} \in \operatorname{Stab}(v)$ for all $e \in \mathcal{F}$.

Let $Q$ be the subgroup generated by $H$ and $\left\{\omega_{q_{\perp}(e)}=\omega_{e}: e \in \mathcal{F}\right\}$. Then $Q \subset \operatorname{Stab}(v)$. By (4.91), $Q$ is generated by $H$ and $\omega_{L^{\perp}}:=\left\{\omega_{x}: x \in L^{\perp}\right\}$. Now in view of (4.94) it is easily verified that the subgroup generated by $Z_{H}\left(\exp \left(\mathcal{A}_{1}\right)\right)$ and $\omega_{L^{\perp}}$ contains $\mathfrak{W}$. The group generated by $\mathfrak{W}$ and $u(L)$ contains $u\left(L^{\perp}\right)$. Therefore

$$
\operatorname{Stab}(v) \supset Q \supset H \cdot \mathfrak{W} u\left(L^{\perp}\right)=Q_{m_{1}+1} .
$$

We will need the following property of $Q_{m_{1}+1}$.

Lemma 4.13. Let $\boldsymbol{x} \in \mathbb{R}^{m_{1}} \backslash\{0\}$. Then there is no closed proper normal subgroup of $Q_{m_{1}+1}$ containing $u(\boldsymbol{x})$.

Proof. Let $N$ be a closed normal subgroup of $Q_{m_{1}+1}$ containing $u(\boldsymbol{x})$. Then $u(\boldsymbol{x})$ belongs to $N \cap H$, which is a normal subgroup of $H$. Since $H \cong \operatorname{SL}\left(m_{1}+1, \mathbb{R}\right)$ is a simple Lie group with finite center, it does not contain an infinite proper normal subgroup. Therefore $H \subset N$. We note that if $\omega \in \mathfrak{W} \cup u\left(L^{\perp}\right)$, then the closure of the group generated by $\omega \exp \left(\mathbb{R} \mathcal{A}_{1}\right) \omega^{-1}$ and $\exp \left(\mathbb{R} \mathcal{A}_{1}\right)$ contains $\omega$. Since $N$ is normal in $Q_{m_{1}+1}$ and $\exp \left(\mathbb{R} \mathcal{A}_{1}\right) \subset H$, we conclude that $\mathfrak{W} \cup u\left(L^{\perp}\right) \subset N$. Therefore $N=Q_{m_{1}+1}$.

\section{INVARIANCE UNDER A UNIPOTENT FLOW}

Our aim is to prove that the measure $\mu$ as in Corollary 3.5 is an algebraic measure. For this purpose, we will first 'stably' modify the measures $\mu_{i}$ and then show that a stable modification of $\mu$ is invariant under a unipotent flow. This will allow us to use Ratner's theorem in our investigation. 
5.1. Stably twisted trajectory. Let $q: \mathbb{R}^{n-1} \rightarrow \mathbb{R}^{m_{k}}$ denote the projection on the span of the first $m_{k}$-coordinates. We suppose that $\varphi: I=[a, b] \rightarrow \mathbb{R}^{n-1}$ satisfies the following condition for all $s \in I$ :

$$
q(\dot{\varphi}(s)) \neq 0 \text {. }
$$

It may be noted that since $\varphi$ is an analytic curve whose image is not contained in a proper affine subspace of $\mathbb{R}^{n-1}, \varphi$ satisfies (5.1) at all but finitely many $s \in I$.

Fix $w_{0} \in \mathbb{R}^{m_{k}} \backslash\{0\}$, and define

$$
W=\left\{u\left(s w_{0}\right): s \in \mathbb{R}\right\} .
$$

Let $Z$ denote the centralizer of $\exp \left(\mathbb{R} \mathcal{A}_{k}\right)$ in $\operatorname{SL}\left(m_{k}+1, \mathbb{R}\right)$. Then $Z$ acts on $\mathbb{R}^{m_{k}}$ via the correspondence $u(z \cdot v)=z u(v) z^{-1}$ for all $z \in Z$ and $v \in \mathbb{R}^{m_{k}}$. This action is transitive on $\mathbb{R}^{m_{k}} \backslash\{0\}$. By (5.1) there exists an analytic function $z: I \rightarrow Z$ such that

$$
z(s) \cdot q(\dot{\varphi}(s))=w_{0}, \quad \forall s \in I,
$$

where $\dot{\varphi}(s)=d \varphi(s) / d s$. In view of 4.1 and (4.3), we set

$$
a_{i}:=a_{\overline{\boldsymbol{\tau}}_{i}}=\exp \left(\mathcal{A}\left(\boldsymbol{t}_{i}\right)\right), \quad \forall i \in \mathbb{N} .
$$

As in (3.1), for any $i \in \mathbb{N}$, let $\lambda_{i}$ be the probability measure on $L / \Lambda$ defined by

$$
\int_{L / \Lambda} f d \lambda_{i}:=\frac{1}{|I|} \int_{s \in I} f\left(z(s) a_{i} u(\varphi(s)) x_{i}\right) d s, \quad \forall f \in \mathrm{C}_{c}(L / \Lambda) .
$$

Since $\left\{a_{\boldsymbol{\tau}_{i}} a_{i}^{-1}: i \in \mathbb{N}\right\}$ and $z(I)$ are contained in compact subsets of $Z$, from Theorem 3.1 we deduce that there exists a probability measure $\lambda$ on $L / \Lambda$ such that, after passing to a subsequence, $\lambda_{i} \rightarrow \lambda$ in the space of probability measures on $L / \Lambda$ with respect to the weak-* topology.

Theorem 5.1. The measure $\lambda$ is $W$-invariant.

Proof. Let $q_{\perp}: \mathbb{R}^{n-1} \rightarrow \mathbb{R}^{n-1-m_{k}}$ denote the projection on the last $\left(n-1-m_{k}\right)$ coordinates. Let $\alpha_{i}=\exp \left(\sum_{j=1}^{n-1} \tau_{i, j}^{\prime}+\tau_{i, m_{k}}^{\prime}\right)$. Then for any $\xi \in \mathbb{R}^{n-1}$,

$$
v=q(\xi)+q_{\perp}(\xi), \quad a_{i} \cdot q(\xi)=\alpha_{i} q(\xi), \quad \text { and } \quad \alpha_{i}^{-1}\left(a_{i} \cdot q_{\perp}(\xi)\right) \stackrel{i \rightarrow \infty}{\longrightarrow} 0 .
$$

Let $t \in \mathbb{R}$. Take any $\epsilon>0$. For $i \in \mathbb{N}$, let $N_{i}:=\left[\epsilon|I| \alpha_{i}\right] \in \mathbb{N}$. Then

$$
\alpha_{i} / N_{i} \stackrel{i \rightarrow \infty}{\longrightarrow}(\epsilon|I|)^{-1} \text { and } \quad \alpha_{i} / N_{i}^{2} \stackrel{i \rightarrow \infty}{\longrightarrow} 0 .
$$

We partition $I=\bigcup_{r=1}^{N_{i}} I_{r}$, where $I_{r}=\left[s_{r}, s_{r+1}\right]$ and $s_{r+1}-s_{r}=|I| / N_{i}$. Let

$$
\begin{aligned}
\psi_{r}(s) & :=\varphi\left(s_{r}\right)+\left(s-s_{0}\right) \dot{\varphi}\left(s_{r}\right), \quad \forall s \in \mathbb{R} ; \text { then } \\
\varphi(s) & =\psi_{r}(s)+\varepsilon_{r}(s) \quad \text { and } \quad \varepsilon_{r}(s)=O\left(N_{i}^{-2}\right), \quad \forall s \in I_{r} .
\end{aligned}
$$

By (5.6) and (5.7), $\sup _{s \in I_{r}}\left\|a_{i} \cdot \varepsilon_{r}(s)\right\| \stackrel{i \rightarrow \infty}{\longrightarrow} 0$. Since $z(\cdot)$ is continuous and bounded, for all large $i$ and $1 \leq r \leq N_{i}$,

$$
\left|f\left(z(s) a_{i} u(\varphi(s)) x_{i}\right)-f\left(z_{r} a_{i} u\left(\psi_{r}(s)\right) x_{i}\right)\right| \leq \epsilon, \quad \forall s \in I_{r},
$$

where $z_{r}=z\left(u\left(s_{r}\right)\right)$; and the same holds for $f^{u\left(t w_{0}\right)}$ in place of $f$, where we define 
$f^{u\left(t w_{0}\right)}(x):=f\left(u\left(t w_{0}\right) x\right)$ for all $x \in L / \Lambda$. Therefore

$$
\left|\int_{L / \Lambda} f(x) d \lambda_{i}(x)-\frac{1}{|I|} \sum_{i=1}^{N_{i}} \int_{I_{r}} f\left(z_{r} a_{i} u\left(\psi_{r}(s)\right) x_{i}\right) d s\right| \leq \epsilon,
$$

and the same for $f^{u\left(t w_{0}\right)}$ in place of $f$.

Next, for any $s \in I_{r}$, by (5.3) and (5.6),

$$
\begin{aligned}
u\left(t w_{0}\right) z_{r} a_{i} u\left(\psi_{r}(s)\right) & =z_{r} u\left(t q\left(\dot{\varphi}\left(s_{r}\right)\right)\right) a_{i} u\left(\psi_{r}(s)\right) \\
& =z_{r} a_{i} u\left(t \alpha_{i}^{-1} q\left(\dot{\varphi}\left(s_{r}\right)\right)\right) u\left(\psi_{r}(s)\right) \\
& =z_{r} a_{i} u\left(-t \alpha_{i}^{-1} q_{\perp}\left(\dot{\varphi}\left(s_{r}\right)\right)\right) u\left(\psi_{r}(s)+t \alpha^{-1} \dot{\varphi}\left(s_{r}\right)\right) \\
& =u\left(z_{r} a_{i} \cdot\left(-t \alpha_{i}^{-1} q_{\perp}\left(\dot{\varphi}\left(s_{r}\right)\right)\right)\right) z_{r} a_{i} u\left(\psi_{r}\left(s+t \alpha_{i}^{-1}\right)\right) .
\end{aligned}
$$

By ([5.6) $\sup _{s \in I_{r}}\left\|z_{r} a_{i} \cdot\left(-t \alpha_{i}^{-1} q_{\perp}\left(\dot{\varphi}\left(s_{r}\right)\right)\right)\right\| \stackrel{i \rightarrow \infty}{\longrightarrow} 0$. Hence for large enough $i$,

$$
\sum_{r=1}^{N_{i}} \int_{I_{r}}\left|f\left(u\left(t w_{0}\right) z_{r} a_{i} \psi_{r}(s) x_{i}\right)-f\left(z_{r} a_{i} \psi_{r}\left(s+t \alpha_{i}^{-1}\right) x_{i}\right)\right| d s \leq \epsilon|I| .
$$

Now by (5.7)

$$
\begin{aligned}
& \sum_{r=1}^{N_{i}}\left|\int_{I_{r}} f\left(z_{r} a_{i} u\left(\psi_{r}(s)\right) x_{i}\right) d s-\int_{I_{r}} f\left(z_{r} a_{i} u\left(\psi_{r}\left(s+t \alpha_{i}^{-1}\right) x_{i}\right)\right) d s\right| \\
& \quad \leq N_{i}\left(2\|f\|_{\infty} t \alpha_{i}^{-1}\right) \stackrel{i \rightarrow \infty}{\longrightarrow} 2 t\|f\|_{\infty} \epsilon|I| .
\end{aligned}
$$

For all large $i$, combining (5.10), (5.12), and (5.13):

$$
\left|\int_{L / \Lambda} f\left(u\left(t w_{0}\right) y\right) d \lambda_{i}(y)-\int_{L / \Lambda} f(y) d \lambda_{i}(y)\right| \leq\left(3+2 t\|f\|_{\infty}\right) \epsilon
$$

Since $\epsilon>0$ is arbitrary, $\lambda$ is $u\left(t w_{0}\right)$-invariant.

\section{RATNER'S THEOREM AND DYNAMICAL BEHAVIOUR OF TRANSLATED TRAJECTORIES NEAR SINGULAR SETS}

Next we will analyze the measure $\lambda$ using Ratner's description of ergodic and invariant measures for unipotent flows.

Let $\mathscr{H}$ be as defined in $\$ 3.1$ and let $W$ be an Ad-unipotent one-parameter subgroup of $G$. For $H \in \mathscr{H}$, define

$$
N(H, W)=\left\{g \in G: g^{-1} W g \subset H\right\} \quad \text { and } \quad S(H, W)=\underset{\substack{F \in \mathscr{H} \\ F \subsetneq H}}{\mathcal{H}_{\mp}} N(F, W) .
$$

Let $\pi: L \rightarrow L / \Lambda$ denote the natural quotient map. By Ratner's theorem [13, as explained in [12, Theorem 2.2]:

Theorem 6.1 (Ratner). Given a $W$-invariant probability measure $\lambda$ on $L / \Lambda$, there exists $H \in \mathscr{H}$ such that

$$
\lambda(\pi(N(H, W)))>0 \quad \text { and } \quad \lambda(\pi(S(H, W)))=0 .
$$

Moreover, almost all $W$-ergodic components of the restriction of $\lambda$ to $\pi(N(H, W))$ are of the form $g \mu_{H}$, where $g \in N(H, W) \backslash S(H, W)$ and $\mu_{H}$ is a finite $H$-invariant measure on $\pi(H) \cong H / H \cap \Lambda$.

In particular if $H$ is a normal subgroup of $L$, then $\lambda$ is $H$-invariant. 
6.1. Algebraic criterion for zero limit measure on singular sets. Similar to the nondivergence criterion given by Proposition 3.3 , the next result provides a criterion for 'non-accumulation on singular sets' in terms of linear actions of groups; it is also referred to as the 'linearization technique'. Let the notation be as in $\$ 3.1$. Let $w_{0} \in \operatorname{Lie}(W) \backslash\{0\}$. Let $\mathscr{A}=\left\{v \in V: v \wedge w_{0}=0\right\}$. Then

$$
N(H, W)=\left\{g \in L: g \cdot p_{H} \in \mathscr{A}\right\} .
$$

The following linearization statement from [19, Proposition 4.4] uses the fact that $\varphi$ is analytic; cf. [14, 6, 12.

Proposition 6.2. Let $C$ be any compact subset of $N(H, W) \backslash S(H, W)$. Let $\epsilon>0$ be given. Then there exists a compact set $\mathcal{D} \subset \mathscr{A}$ such that given any neighbourhood $\Phi$ of $\mathcal{D}$ in $V$, there exists a neighbourhood $\mathcal{O}$ of $\pi(C)$ in $L / \Lambda$ such that for any $h_{1}, h_{2} \in L$ and a subinterval $J \subset I$, one of the following holds:

(a) There exists $\gamma \in \Lambda$ such that $\left(h_{1} z(s) u(\varphi(s)) h_{2} \gamma\right) p_{H} \in \Phi, \forall s \in J$.

(b) $\left|\left\{s \in J: \pi\left(h_{1} z(s) u(\varphi(s)) h_{2}\right) \in \mathcal{O}\right\}\right| \leq \epsilon|J|$.

Just as in the proof of Theorem 3.1 we will apply this criterion to obtain an algebraic condition leading to the hypothesis of Corollary 4.10

Theorem 6.3. Suppose that there is no proper closed subgroup $H$ of $L$ containing $\rho\left(Q_{m_{1}+1}\right)$ such that the orbit $H x_{0}$ is closed and admits a finite $H$-invariant measure. Let $\left\{\lambda_{i}\right\}_{i=1}^{\infty}$ be the sequence of measures as defined by (5.5). Then $\lambda_{i} \stackrel{i \rightarrow \infty}{\longrightarrow} \lambda$ in the space of probability measures on $L / \Lambda$ and $\lambda$ is L-invariant.

Proof. Earlier, using Theorem 3.1 we have shown that after passing to a subsequence, $\lambda_{i} \rightarrow \lambda$ in the space of probability measures on $L / \Lambda$, and by Theorem [5.1. $\lambda$ is invariant under the Ad-unipotent one-parameter subgroup $W$. In order to complete the proof, it is enough to show that any such limiting measure $\lambda$ is $L$-invariant. For notational convenience we will identify any $g \in G$ with $\rho(g) \in L$.

By Theorem 6.1 there exists $H \in \mathscr{H}$ such that

$$
\lambda(\pi(N(H, W)))>0 \quad \text { and } \quad \lambda(\pi(S(H, W)))=0 .
$$

Let $C$ be a compact subset of $N(H, W) \backslash S(H, W)$ such that $\lambda(C)>\epsilon$ for some $\epsilon>0$. Let $g_{i} \stackrel{i \rightarrow \infty}{\longrightarrow} g_{0}$ be a sequence in $G$ such that $x_{0}=\pi\left(g_{0}\right)$ and $x_{i}=\pi\left(g_{i}\right)$ for all $i$. Then given any neighbourhood $\mathcal{O}$ of $\pi(C)$ in $L / \Lambda$, there exists $i_{0}>0$ such that for all $i \geq i_{0}$, we have $\lambda_{i}(\mathcal{O})>\epsilon$ and hence

$$
\frac{1}{|I|}\left|\left\{s \in I: z(s) a_{i} u(\varphi(s)) x_{i}=\pi\left(a_{i} z(s) u(\varphi(s)) g_{i}\right) \in \mathcal{O}\right\}\right|>\epsilon .
$$

Let $\mathcal{D} \subset \mathscr{A}$ be as in the statement of Proposition 6.2 Choose any compact neighbourhood $\Phi$ of $\mathcal{D}$ in $V$. Then there exists a neighbourhood $\mathcal{O}$ of $\pi(C)$ in $L / \Lambda$ such that one of the statements (a) or (b) of Proposition 6.2 holds for $J=I$ and any $h_{1}=a_{i}$ and $h_{2}=g_{i}$. For any $i>i_{0}$, (a) cannot hold due to (6.5), and hence (b) must hold; that is, there exists $\gamma_{i} \in \Lambda$ such that

$$
\left(z(s) a_{i} u(\varphi(s)) g_{i} \gamma_{i}\right) p_{H}=\left(a_{i} z(s) u(\varphi(s)) g_{i} \gamma_{i}\right) p_{H} \in \Phi, \quad \forall s \in I .
$$

Let $\Phi_{1}=\left\{z(s)^{-1}: s \in I\right\} \Phi$. Then $\Phi_{1}$ is contained in a compact subset of $V$, and the following holds:

$$
a_{i} u(\varphi(s))\left(g_{i} \gamma_{i}\right) p_{H} \in \Phi_{1}, \quad \forall s \in I, \forall i>i_{0} .
$$


Let $\|\cdot\|$ be a norm on $V$. First suppose that after passing to a subsequence,

$$
r_{i}:=\left\|\gamma_{i} p_{H}\right\| \rightarrow \infty \quad \text { as } i \rightarrow \infty \text {. }
$$

Then $v_{i}:=\gamma_{i} p_{H} / r_{i} \stackrel{i \rightarrow \infty}{\longrightarrow} v$ for some $v \in V,\|v\|=1$. Let $R=\sup \left\{\|w\|: w \in \Phi_{1}\right\}$. Then by 6.7

$$
a_{i} u(\varphi(s)) g_{i} v_{i} \leq R / r_{i}, \quad \forall s \in I, \forall i>i_{0} .
$$

Since $R / r_{i} \stackrel{i \rightarrow \infty}{\longrightarrow} 0$ and $g_{i} v_{i} \stackrel{i \rightarrow \infty}{\longrightarrow} g_{0} v$, we conclude that

$$
u(\varphi(s)) g_{0} v_{0} \subset V^{-}(\mathcal{T}), \quad \forall s \in I .
$$

Since there exists a finite set $F \subset I$ such that $\mathcal{B}=\{\varphi(s): s \in F\}$ is an affine basis of $\mathbb{R}^{n-1}$, (6.10) satisfies the condition (4.64) of Corollary 4.10 but contradicts its conclusion (4.65). Thus (6.8) fails to hold after passing to a subsequence. Therefore the set $\left\{\gamma_{i} p_{H}: i \in \mathbb{N}\right\}$ is bounded. It is discrete by Proposition 3.2. Hence it is a finite set. Therefore by passing to a subsequence, there exists $\gamma \in \Gamma$ such that

$$
\gamma_{i} p_{H}=\gamma p_{H}, \quad \forall i \in \mathbb{N} .
$$

Therefore by (6.7) we get

$$
a_{i} u(\varphi(s)) g_{i}\left(\gamma p_{H}\right) \subset \Phi_{1}, \quad \forall s \in I, \forall i \in \mathbb{N} .
$$

Let $\pi_{+}^{\mathcal{T}}: V \rightarrow V^{+}(\mathcal{T})$ be the projection parallel to $V^{0}(\mathcal{T})+V^{-}(\mathcal{T})$. First suppose that $\pi_{+}^{\mathcal{T}}\left(u(\varphi(s)) g_{0} \gamma p_{H}\right) \neq 0$ for some $s_{0} \in I$. Then there exists $c>0$ and $i_{1} \in \mathbb{N}$ such that $\left\|\pi_{+}^{\mathcal{T}}\left(u\left(\varphi\left(s_{0}\right)\right) g_{i} \gamma p_{H}\right)\right\| \geq c$ for all $i \geq i_{1}$. But then $\left\|a_{i} u\left(\varphi\left(s_{0}\right) g_{i} \gamma p_{H}\right)\right\| \stackrel{i \rightarrow \infty}{\longrightarrow}$ $\infty$, which contradicts (6.12). Therefore

$$
u(\varphi(s))\left(g_{0} \gamma p_{H}\right) \subset V^{0}(\mathcal{T}) \oplus V^{-}(\mathcal{T}), \quad \forall s \in I .
$$

Therefore by Proposition 4.12, $Q_{m_{1}+1}$ stabilizes $g_{0} \gamma p_{H}$. By (3.2),

$$
g_{0}^{-1} Q_{m_{1}+1} g_{0} \subset \mathrm{N}_{\mathrm{L}}^{1}(H)=\operatorname{Stab}_{L}\left(\gamma p_{H}\right) .
$$

Since $\Lambda p_{H}$ is discrete, $\Lambda \mathrm{N}_{\mathrm{L}}^{1}(H)$ is a closed subset of $L$. Hence $\pi\left(\mathrm{N}_{\mathrm{L}}^{1}(H)\right)$ is closed in $L / \Lambda$. By [16, Theorem 2.3] there exists a closed subgroup $H_{1}$ of $\mathrm{N}_{\mathrm{L}}^{1}(H)$ containing all Ad-unipotent one-parameter subgroups of $L$ contained in $\mathrm{N}_{\mathrm{L}}^{1}(H)$ such that $H_{1} \cap \Lambda$ is a lattice in $H_{1}$ and $\pi\left(H_{1}\right)$ is closed. Since $Q_{m_{1}+1}$ is generated by unipotent one-parameter subgroups of $\mathrm{SL}(n, \mathbb{R})$, by (6.14), $g_{0}^{-1} Q_{m_{1}+1} g_{0} \subset H_{1}$. Thus $Q_{m_{1}+1} \subset g_{0} H_{1} g_{0}^{-1}$ and $\left(g_{0} H_{1} g_{0}^{-1}\right) x_{0}=g_{0} \pi\left(H_{1}\right)$ is closed and admits a finite $g_{0} \mathrm{Hg}_{0}^{-1}$-invariant measure. Hence by the hypothesis of the theorem, $g_{0} H_{1} g_{0}^{-1}=L$. Therefore $H$ is a normal subgroup of $L$. Therefore by Theorem $6.1, \lambda$ is $H$-invariant.

By (6.4) there exists $g \in N(H, W) \neq \emptyset$. Then $W \subset g H^{-1}=H$. Thus $W \subset Q_{m_{1}+1} \cap H$, which is a normal subgroup of $Q_{m_{1}+1} \cap H$. Hence by Lemma 4.13 we have $Q_{m_{1}+1} \subset H$. Since $H x_{0}=\pi\left(H g_{0}\right)=g_{0} \pi(H)$ is closed and admits a finite $H$-invariant measure, by our hypothesis $H=L$. Therefore $\lambda$ is $L$-invariant.

Proof of Theorem 1.2. Let the notation be as in $\$ 4.1$. Then

$$
\boldsymbol{\tau}_{i}-\overline{\boldsymbol{\tau}}_{i} \stackrel{i \rightarrow \infty}{\longrightarrow} \tilde{\boldsymbol{\tau}}_{0}:=(\tau(1), \ldots, \tau(n-1)) \in \mathbb{R}^{n-1}, \quad \text { as } i \rightarrow \infty .
$$

Therefore $a_{\boldsymbol{\tau}_{i}} a_{\overline{\boldsymbol{\tau}}_{i}}^{-1} \stackrel{i \rightarrow \infty}{\longrightarrow} a_{\tilde{\boldsymbol{\tau}}_{0}}$. Hence in $G / Q_{m_{1}+1}$,

$$
a_{\boldsymbol{\tau}_{i}} Q_{m_{1}+1} \stackrel{i \rightarrow \infty}{\longrightarrow} a_{\tilde{\boldsymbol{\tau}}_{0}} Q_{m_{1}+1}=a_{\boldsymbol{\tau}_{0}} Q_{m_{1}+1} .
$$

Therefore to prove (1.6), it is enough to prove the theorem in the case of $\tau_{i}=\bar{\tau}_{i}$. 
We put $x_{i}=x_{0}$ for all $i$. As noted earlier, there exists a smallest closed subgroup $H$ of $L$ containing $\rho\left(Q_{m_{1}+1}\right)$ such that the orbit $H x_{0}$ is closed and admits a finite $H$-invariant measure. Therefore without loss of generality we may replace $L$ by $H$. Now to complete the proof of the theorem, we only need to prove that $\mu$ is $L$-invariant.

Since $\varphi$ is analytic, the condition (5.3) fails to hold only for finitely many points, and it is straightforward to reduce the proof of the theorem to the case where (5.3) holds for all $s \in I$. Now the difference between $\mu_{i}$ and $\lambda_{i}$ is only through $\{z(s): s \in I\}$. Given $\epsilon>0$, there exists $\delta>0$ such that if $J=\left[s_{1}, s_{2}\right] \subset I$ and $0<s_{2}-s_{1}<\delta$, then $\left|f\left(z\left(s_{1}\right)^{-1} z(s) x\right)-f(x)\right| \leq \epsilon$ for all $s \in J$ and $x \in L / \Lambda$. We define $\lambda_{i}^{J}$ by putting $J$ in place of $I$ in (5.5); we define $\mu_{i}^{J}$ similarly. Then by Theorem 6.3, $\lambda_{i}^{J} \rightarrow \lambda_{L}$, where $\lambda_{L}$ is the unique $L$-invariant probability measure on $L / \Lambda$. Since $a_{i} z(s)=z(s) a_{i}$, we deduce that

$$
\left|\int f d \mu_{i}^{J}-\int f\left(z\left(s_{1}\right)^{-1} x\right) d \lambda_{L}(x)\right| \leq \epsilon
$$

for all large $i$. Since $\lambda_{L}$ is $z\left(s_{1}\right)$-invariant, the second integral is the same as $\int f d \lambda_{L}$. Now partitioning $I$ into finitely many $J$ 's with $|J| \leq \delta$, we deduce

$$
\left|\int f d \mu_{i}-\int f d \lambda_{L}\right| \leq \epsilon
$$

for all large $i$. Thus $\mu_{i} \stackrel{i \rightarrow \infty}{\longrightarrow} \lambda_{L}$.

Proof of Theorem 1.10. The above proof applies to this case also. Here we are given that $x_{i} \rightarrow x_{0}$ is a convergent sequence and there is no proper closed subgroup $H$ of $G$ containing $\rho\left(Q_{m_{1}+1}\right)$ such that $H x_{0}$ is closed and admits a finite $H$-invariant measure. Therefore there is no need to replace $H$ by $L$ as in the above proof.

The proof of Theorem 1.11 can be obtained by combining the ideas of the proof of Theorem 6.3. Proposition 4.2, and the general strategy of the proof of [6, Theorem 3].

\section{ACKNOWLEDGMENT}

I am very thankful to Shahar Mozes and Elon Lindenstrauss for several helpful discussions. I would also like to thank Dmitry Kleinbock for useful suggestions. Thanks are due to the referee for many remarks leading to improved presentation and clearer proofs.

\section{REFERENCES}

[1] R. C. Baker. Dirichlet's theorem on Diophantine approximation. Math. Proc. Cambridge Philos. Soc., 83(1):37-59, 1978. MR0485713 (58:5535)

[2] Y. Bugeaud. Approximation by algebraic integers and Hausdorff dimension. J. London Math. Soc. (2), 65(3):547-559, 2002. MR1895732 (2003d:11110)

[3] J. W. S. Cassels. An introduction to the geometry of numbers. Die Grundlehren der mathematischen Wissenschaften, Band 99. Springer-Verlag, Berlin-New York, 1971. viii+344 pp. MR0306130 (46:5257)

[4] S. G. Dani. Divergent trajectories of flows on homogeneous spaces and Diophantine approximation. J. Reine Angew. Math., 359:55-89, 1985. MR794799 (87g:58110a)

[5] S. G. Dani and G. A. Margulis. Asymptotic behaviour of trajectories of unipotent flows on homogeneous spaces. Proc. Indian Acad. Sci. Math. Sci., 101(1):1-17, 1991. MR1101994 (92g:22027) 
[6] S. G. Dani and G. A. Margulis. Limit distributions of orbits of unipotent flows and values of quadratic forms. In I. M. Gelfand Seminar, pages 91-137. Amer. Math. Soc., Providence, RI, 1993. MR1237827 (95b:22024)

[7] H. Davenport and W. M. Schmidt Dirichlet's theorem on diophantine approximation. II. Acta Arith., 16:413-424, 1969/1970. MR0279040(43:4766)

[8] H. Davenport and W. M. Schmidt. Dirichlet's theorem on diophantine approximation. Symposia Mathematica, Vol. IV (INDAM, Rome, 1968/69), pp. 113-132, Academic Press, London, 1970. MR0272722(42:7603)

[9] M. M. Dodson, B. P. Rynne, J. A. Vickers. Dirichlet's theorem and Diophantine approximation on manifolds. J. Number Theory, 36(1):85-88, 1990. MR.1068674 (91k:11063)

[10] D. Y. Kleinbock and G. A. Margulis. Flows on homogeneous spaces and Diophantine approximation on manifolds. Ann. of Math. (2), 148(1):339-360, 1998. MR1652916 (99j:11083)

[11] Dmitry Kleinbock and Barak Weiss. Dirichlet's theorem on Diophantine approximation and homogeneous flows. Journal of Modern Dynamics (JMD), 2(1):43-62, 2008. MR2366229 (2008k:11078)

[12] Shahar Mozes and Nimish A. Shah. On the space of ergodic invariant measures of unipotent flows. Ergodic Theory Dynam. Systems, 15(1):149-159, 1995. MR,1314973 (95k:58096)

[13] Marina Ratner. On Raghunathan's measure conjecture. Ann. of Math. (2), 134(3):545-607, 1991. MR $1135878(93 a: 22009)$

[14] Marina Ratner. Raghunathan's topological conjecture and distributions of unipotent flows. Duke Math. J., 63(1):235-280, 1991. MR1106945 (93f:22012)

[15] Wolfgang M. Schmidt. Diophantine approximation. Lecture Notes in Mathematics, 785, Springer, Berlin, 1980, x+299 pp. MR $568710(81 \mathrm{j}: 10038)$

[16] Nimish A. Shah. Uniformly distributed orbits of certain flows on homogeneous spaces. Math. Ann., 289(2):315-334, 1991. MR 1092178 (93d:22010)

[17] Nimish A. Shah. Limit distributions of polynomial trajectories on homogeneous spaces. Duke Math. J., 75(3):711-732, 1994. MR1291701(95j:22022)

[18] Nimish A. Shah. Limit distributions of expanding translates of certain orbits on homogeneous spaces. Proc. Indian Acad. Sci. (Math. Sci.), 106:105-125, 1996. MR1403756 (98b:22024)

[19] Nimish A. Shah. Limiting distributions of curves under geodesic flow on hyperbolic manifolds. Duke Math. J., 148(2):251-279, 2009. MR2524496

[20] Nimish A. Shah. Equidistribution of expanding translates of curves and Dirichlet's theorem on Diophantine approximation. Invent. Math., 177:509-532, 2009. MR2534098

Tata Institute of Fundamental Research, Mumbai 400005, India Current address: Department of Mathematics, Ohio State University, Columbus, Ohio 43210 E-mail address: nimish@math.tifr.res.in; shah@math.osu.edu 